\title{
Review of the clinical pharmacokinetics of artesunate and its active metabolite dihydroartemisinin following intravenous, intramuscular, oral or rectal administration
}

Carrie A Morris', Stephan Duparc ${ }^{2}$, Isabelle Borghini-Fuhrer ${ }^{2}$, Donald Jung ${ }^{3}$, Chang-Sik Shin ${ }^{4}$ and Lawrence Fleckenstein ${ }^{1 *}$

\begin{abstract}
Artesunate (AS) is a clinically versatile artemisinin derivative utilized for the treatment of mild to severe malaria infection. Given the therapeutic significance of AS and the necessity of appropriate AS dosing, substantial research has been performed investigating the pharmacokinetics of AS and its active metabolite dihydroartemisinin (DHA). In this article, a comprehensive review is presented of AS clinical pharmacokinetics following administration of AS by the intravenous (IV), intramuscular (IM), oral or rectal routes. Intravenous AS is associated with high initial AS concentrations which subsequently decline rapidly, with typical AS half-life estimates of less than 15 minutes. AS clearance and volume estimates average $2-3 \mathrm{~L} / \mathrm{kg} / \mathrm{hr}$ and $0.1-0.3 \mathrm{~L} / \mathrm{kg}$, respectively. DHA concentrations peak within 25 minutes post-dose, and DHA is eliminated with a half-life of 30 - 60 minutes. DHA clearance and volume average between $0.5-1.5 \mathrm{~L} / \mathrm{kg} / \mathrm{hr}$ and $0.5-1.0 \mathrm{~L} / \mathrm{kg}$, respectively. Compared to IV administration, IM administration produces lower peaks, longer half-life values, and higher volumes of distribution for AS, as well as delayed peaks for DHA; other parameters are generally similar due to the high bioavailability, assessed by exposure to DHA, associated with IM AS administration (> 86\%). Similarly high bioavailability of DHA (> 80\%) is associated with oral administration. Following oral AS, peak AS concentrations (Cmax) are achieved within one hour, and AS is eliminated with a half-life of 20 - 45 minutes. DHA Cmax values are observed within two hours post-dose; DHA half-life values average $0.5-1.5$ hours. AUC values reported for AS are often substantially lower than those reported for DHA following oral AS administration. Rectal AS administration yields pharmacokinetic results similar to those obtained from oral administration, with the exceptions of delayed AS Cmax and longer AS half-life. Drug interaction studies conducted with oral AS suggest that AS does not appreciably alter the pharmacokinetics of atovaquone/proguanil, chlorproguanil/dapsone, or sulphadoxine/pyrimethamine, and mefloquine and pyronaridine do not alter the pharmacokinetics of DHA. Finally, there is evidence suggesting that the pharmacokinetics of AS and/or DHA following AS administration may be altered by pregnancy and by acute malaria infection, but further investigation would be required to define those alterations precisely.
\end{abstract}

\section{Background}

Derivatives of the naturally occurring endoperoxide antimalarial artemisinin form the foundation of the current global treatment approach for Plasmodium falciparum malaria. These derivatives, including artesunate (AS),

\footnotetext{
* Correspondence: I-fleckenstein@uiowa.edu

'Pharmaceutical Sciences and Experimental Therapeutics, University of lowa College of Pharmacy, 115 South Grand Avenue, lowa City, IA 52242, USA Full list of author information is available at the end of the article

artemether and dihydroartemisinin, produce more profound reductions in parasitaemia and more rapid symptom relief than agents from any other anti-malarial class. Of these derivatives, AS is the most therapeutically versatile agent. As with the other derivatives, AS can be administered orally for the treatment of uncomplicated malaria. Specifically, artemisinin-based combination therapies containing AS partnered with longer acting anti-malarial agents, such as mefloquine or 
sulphadoxine-pyrimethamine, are extensively utilized for oral treatment of uncomplicated falciparum malaria [1]. Among the artemisinin derivatives, however, only AS displays sufficient water solubility to be administered intravenously; per the World Health Organization treatment guidelines, intravenous AS is the preferred therapy for severe malaria infection in both adult and paediatric patients [1]. AS can also be administered intramuscularly or rectally, with AS suppositories for rectal administration representing a means of initiating treatment of severe malaria before patients are referred to distant facilities for intravenous therapy.

Given the therapeutic significance and versatility of AS, and the necessity of appropriate dosing to avoid suboptimal efficacy or encouragement of resistance, research defining the pharmacokinetics (PK) of AS, and its active metabolite dihydroartemisinin (DHA), is of substantial clinical relevance. The intent of this review is to examine clinical pharmacokinetic findings of AS and DHA following AS administration by the intravenous (IV), intramuscular (IM), oral and rectal routes. To this end, an extensive literature search was conducted utilizing the PubMed database and the bibliographies of identified articles in order to locate AS clinical pharmacokinetic studies in which parameters for AS and/or DHA are reported. The PubMed database was searched using combinations of the following search terms: artesunate, dihydroartemisinin, artemisinin, and pharmacokinetics. Conference abstracts and non-English language articles were not considered for inclusion in the review.

To facilitate comparison of results among various studies, units for these parameters were converted, as necessary, to a uniform scale as noted in the tables included in this review. Additionally, individual PK analyses and population pharmacokinetic (PopPK) analyses are described separately for each route of administration, where applicable, to enable adequate description of the findings from each analysis method.

\section{Sources of variation introduced by study methodology}

Multiple factors complicate comparison and summation of AS/DHA pharmacokinetic findings across multiple studies, including differences in assay sensitivities, sampling schedules, and choice of anticoagulant for blood sample collection. Differences in sampling schedules are of particular importance in comparisons of AS pharmacokinetic parameters; a relative lack of sampling points in the early post-dose period can result in much of subjects' AS exposure being missed. With regard to choice of anticoagulant, if fluoride-oxalate, rather than heparin, is included as the anticoagulant in blood sample collection tubes, ex vivo plasma esterase degradation of AS to DHA is greatly inhibited [2]. This inhibition allows for greater preservation of a subject's AS concentrations at the time of blood sample collection. However, fluorideoxalate may also result in greater erythrocyte shrinkage than heparin, and therefore increased plasma volume [2]. Given these sources of variation, differences in pharmacokinetic findings among the studies described in this review cannot necessarily be regarded as solely related to whatever specific demographic or clinical features characterize the study subjects.

\section{Artesunate and DHA protein binding}

Binding of AS to human plasma proteins has been investigated utilizing equilibrium dialysis with $\left[{ }^{14} \mathrm{C}\right]$ artesunate. AS was determined to be $75 \%$ protein bound at plasma concentrations less than $125 \mathrm{ng} / \mathrm{mL}$, and $62 \%$ protein bound at higher concentrations [3]. DHA plasma protein binding, when measured by similar means, was determined to be $82 \%$ at plasma concentrations less than $25 \mathrm{ng} / \mathrm{mL}$ and $66 \%$ at higher concentrations [4]. DHA percent bound was also assessed by ultrafiltration in patients with malaria infection (falciparum or vivax), Vietnamese healthy volunteers, and Caucasian volunteers and determined to be $93 \%, 88 \%$, and $91 \%$, respectively [5]. However, as AS and DHA are both high extraction ratio drugs [6], any alterations in patients' protein binding capacity would not be expected to produce clinically relevant changes in the clearance of either agent.

\section{Intravenous administration: artesunate pharmacokinetics}

The pharmacokinetic results of the identified studies in which intravenous AS PK were assessed are presented in Tables 1 and 2[7-15]; both AS and DHA PK were described in eight of these studies, with only DHA PK described in the ninth [15]. In clinical settings, IV AS is administered as a bolus injection [16]. As the identified studies replicated this administration method, very high AS maximum concentrations ( $\mathrm{Cmax}$ ) were observed. AS is metabolized through esterase-catalyzed hydrolysis to yield its active metabolite, DHA [17]; this conversion occurs quickly following IV AS administration as indicated by the rapid decline in AS concentrations in the early post-dose period. In six of the eight studies in which AS PK were assessed, average AS half-life following IV AS was determined to be less than five minutes in at least one study cohort. In all of the studies, average AS half-life was determined to be less than fifteen minutes. Finally, per the findings of Li et al [11], AS was determined to display dose linearity following IV administration across a dosage range of $0.5-8 \mathrm{mg} / \mathrm{kg}$.

Examination of the AS clearance and volume estimates summarized in Table 1 indicates that the parameters obtained by Newton et al [13] are dissimilar from the parameters obtained in other studies, likely 
Table 1 Summary of AS pharmacokinetic results following IV AS administration

\begin{tabular}{|c|c|c|c|c|c|c|}
\hline Ref. & Subjects \& Regimen & $\mathrm{Cmax}(\mathrm{ng} / \mathrm{mL})$ & $\begin{array}{l}\text { Clearance } \\
(\mathrm{L} / \mathrm{kg} / \mathrm{hr})\end{array}$ & Volume (L/kg) & $\begin{array}{l}\text { Half-life } \\
(\min )\end{array}$ & $\begin{array}{l}\text { AUC } \\
\left(\mathrm{ng} \mathrm{g}^{*} \mathrm{hr} / \mathrm{mL}\right)\end{array}$ \\
\hline $\begin{array}{l}\text { [7] } \\
\text { Batty, Le } \\
\text { et al, } 1998\end{array}$ & $\begin{array}{l}12 \text { Vietnamese adults with vivax malaria } \\
120 \text { mg IV AS over } 2 \text { min }\end{array}$ & $13685 t^{\mathrm{a}}$ & 3.01 & 0.16 & 2.19 & $876+$ \\
\hline $\begin{array}{l}{[9]} \\
\text { Binh et al, } \\
2001\end{array}$ & $\begin{array}{l}17 \text { healthy Vietnamese volunteers; subjects randomized } \\
\text { into two groups, both receiving: } \\
120 \text { mg IV AS over } 2 \text { min }\end{array}$ & & $3.0 ; 2.2$ & $0.19 ; 0.16$ & $2.6 ; 3.3$ & $846 ; 1269+$ \\
\hline $\begin{array}{l}{[10]} \\
\text { llett et al, } \\
2002\end{array}$ & $\begin{array}{l}23 \text { Vietnamese adults with uncomplicated falciparum } \\
\text { malaria; subjects randomized into two groups, both } \\
\text { receiving: } \\
120 \text { mg IV AS over } 2 \text { min }\end{array}$ & $\begin{array}{l}16146 \\
16530+\end{array}$ & $2.8 ; 2.1$ & 0.22 & 3.2 & $1038 ; 1230+$ \\
\hline $\begin{array}{l}{[11]} \\
\text { Li et al, } \\
2009\end{array}$ & $\begin{array}{l}30 \text { healthy volunteers } \\
0.5,1,2,4 \text {, or } 8 \mathrm{mg} / \mathrm{kg} \text { IV AS over } 2 \mathrm{~min}\end{array}$ & $\begin{array}{l}4797 ; 6128 \\
19420 ; 36100 \\
83340\end{array}$ & $\begin{array}{l}1.3 ; 18 ; 1.3 ; \\
1.4 ; 1.6+\end{array}$ & $\begin{array}{l}\text { Vss: 0.092; 0.187; } \\
0.106 ; 0.109 ; \\
0.165+\end{array}$ & $\begin{array}{l}7.2 ; 8.4 ; \\
14.4 ; 9.0 ; \\
12.6+\end{array}$ & $\begin{array}{l}386 ; 593 ; \\
1595 ; 3038 ; \\
6994 \dagger\end{array}$ \\
\hline $\begin{array}{l}{[12]} \\
\text { Nealon et } \\
a l, 2002\end{array}$ & $\begin{array}{l}28 \text { paediatric Gabonese patients with severe malaria } \\
\text { randomized into two groups } \\
\text { Group 1: } 2.4 \mathrm{mg} / \mathrm{kg} \text { IV AS } \\
\text { Group 2: } 1.2 \mathrm{mg} / \mathrm{kg} \text { IV AS }\end{array}$ & $\begin{array}{l}\text { Group 1: } \\
29677^{\mathrm{a}} \\
\text { Group 2: } \\
15369^{\mathrm{a}}\end{array}$ & $\begin{array}{l}\text { Group 1: } \\
3.12 t^{\mathrm{a}} \\
\text { Group 2: } \\
4.26 t^{\mathrm{a}}\end{array}$ & $\begin{array}{l}\text { Group } 1 \text { Vss: } \\
0.17+^{\mathrm{a}} \\
\text { Group } 2 \text { Vss: } \\
0.44 \dagger^{\mathrm{a}}\end{array}$ & $\begin{array}{l}\text { Group 1: } \\
1.5^{\mathrm{a}} \\
\text { Group } \\
2: 11.5^{\mathrm{a}}\end{array}$ & $\begin{array}{l}\text { Group 1: } \\
1042 t^{a} \\
\text { Group 2: } \\
555 t^{\mathrm{a}}\end{array}$ \\
\hline $\begin{array}{l}13] \\
\text { Newton et } \\
\text { al, } 2006 \\
\end{array}$ & $\begin{array}{l}17 \text { adults with severe falciparum malaria in Thailand } \\
2.4 \mathrm{mg} / \mathrm{kg} \text { IV AS over } 2 \mathrm{~min} \\
{[2.1(1.4-2.8 \mathrm{mg} / \mathrm{kg})]}\end{array}$ & $130^{+} t^{a}$ & $64^{\mathrm{a}}$ & $\begin{array}{l}\text { Vss: } \\
15.2^{\mathrm{a}}\end{array}$ & $13.2^{+} t^{a}$ & $49.2 t^{a}$ \\
\hline $\begin{array}{l}\text { [8] } \\
\text { Batty. Thu } \\
\text { et al, } 1998 \\
\end{array}$ & $\begin{array}{l}26 \text { adult uncomplicated falciparum malaria patients in } \\
\text { Vietnam } \\
120 \text { mg IV AS over } 2 \text { min }\end{array}$ & & $2.33^{\mathrm{a}}$ & $0.140^{a}$ & 2.73 & $1146+$ \\
\hline $\begin{array}{l}\text { [14] } \\
\text { Davis et } \\
\text { al, } 2001\end{array}$ & $\begin{array}{l}30 \text { parasitaemic adults with falciparum malaria } \\
\text { Group 1: } 12 \text { with complications (120 mg IV AS over } 2 \\
\text { min) } \\
\text { Group 2: } 8 \text { without complications } \\
\text { (120 mg IV AS over } 2 \text { min) } \\
\text { Group 3: } 10 \text { with moderately severe complications } \\
\text { (240 mg IV AS infused over } 4 \text { hours; samples taken after } \\
\text { infusion discontinued) }\end{array}$ & & $\begin{array}{l}\text { Group 1: } \\
1.63 \\
\text { Group 2: } \\
2.49 \\
\text { Group 3: } \\
3.07\end{array}$ & $\begin{array}{l}\text { Group 1: } \\
0.08 \\
\text { Group 2: } \\
0.24 \\
\text { Group 3: } \\
0.23\end{array}$ & $\begin{array}{l}\text { Group 1: } \\
2.3 \\
\text { Group 2: } \\
4.3 \\
\text { Group 3: } \\
3.2\end{array}$ & \\
\hline
\end{tabular}

Values given as mean unless otherwise specified.

tUnits converted to uniform scale +The authors note that Cmax is likely underestimated and half-life overestimated due to the lack of usable data from six patients with extremely rapid AS elimination. a.Median

due to lack of sampling prior to 15 minutes post-dose; the other summarized studies included a sampling point at or before five minutes. The remaining seven studies have more consistent clearance and volume estimates, with averages ranging from $1.3-4.26 \mathrm{~L} / \mathrm{kg} / \mathrm{hr}$ and 0.08 $0.44 \mathrm{~L} / \mathrm{kg}$, respectively. A majority of the estimates range between $2-3 \mathrm{~L} / \mathrm{kg} / \mathrm{hr}$ for clearance and $0.1-0.3$ $\mathrm{L} / \mathrm{kg}$ for volume.

\section{Intravenous administration: DHA pharmacokinetics}

In all of identified IV AS studies, the time to maximum concentration (Tmax) of DHA following IV AS administration was less than 25 minutes. DHA metabolism occurs through conjugation of DHA by the UDP-glucuronosyltransferase system, with UGT1A9 and UGT2B7 being the primary responsible isoforms [18]. This DHA elimination process occurs somewhat more slowly than the esterase-catalyzed AS elimination, with average halflife estimates for DHA following IV AS administration ranging from 18 minutes to 2.14 hours, with eight of the nine studies citing average estimates for all study cohorts of less than 65 minutes and the majority of estimates falling between $30-60$ minutes. DHA apparent clearance $(\mathrm{Cl} / \mathrm{F})$ and volume of distribution $(\mathrm{V} / \mathrm{F})$ averages ranged from $0.48-5.6 \mathrm{~L} / \mathrm{kg} / \mathrm{hr}$ and $0.55-$ $2.403 \mathrm{~L} / \mathrm{kg}$, with a majority of the estimates averaging $0.5-1.5 \mathrm{~L} / \mathrm{kg} / \mathrm{hr}$ for clearance and $0.5-1.0 \mathrm{~L} / \mathrm{kg}$ for volume. As with AS, DHA displayed dose-linearity across an IV AS dosage range of $0.5-8 \mathrm{mg} / \mathrm{kg}$ [11].

\section{Intravenous administration: bioassay results}

The anti-malarial bioassay method for determination of AS/DHA plasma concentrations provides values in DHA equivalents reflecting the contribution of both AS and DHA present in the sample. An assessment of antimalarial bioactivity in patients with acute uncomplicated falciparum malaria administered $2 \mathrm{mg} / \mathrm{kg}$ IV AS yielded estimates for half-life, volume of distribution at steady state (Vss), and clearance of 0.73 hours, $0.61 \mathrm{~L} / \mathrm{kg}$, and $0.83 \mathrm{~L} / \mathrm{kg} / \mathrm{hr}$, respectively [19]. Bioassay data were also used to compute the pharmacokinetic parameters following administration of $2.4 \mathrm{mg} / \mathrm{kg}$ IV AS to 
Table 2 Summary of DHA pharmacokinetic results following IV AS administration

\begin{tabular}{|c|c|c|c|c|c|c|c|}
\hline Ref. & Subjects \& Regimen & $\begin{array}{l}\text { Cmax } \\
(\mathrm{ng} / \mathrm{mL})\end{array}$ & $\begin{array}{l}\text { Tmax } \\
(\min )\end{array}$ & $\begin{array}{l}\text { Clearance } \\
(\mathrm{L} / \mathrm{kg} / \mathrm{hr})\end{array}$ & $\begin{array}{l}\text { Volume } \\
\text { (L/kg) }\end{array}$ & $\begin{array}{l}\text { Half-life } \\
\text { (min) }\end{array}$ & $\begin{array}{l}\text { AUC } \\
\text { (ng*hr/mL) }\end{array}$ \\
\hline $\begin{array}{l}7] \\
\text { Batty, Le } \\
\text { et al, } \\
1998\end{array}$ & $\begin{array}{l}12 \text { Vietnamese adults with vivax malaria } \\
120 \text { mg IV AS over } 2 \text { min }\end{array}$ & $2192 t^{a}$ & $8^{a}$ & 1.10 & 0.92 & 36.7 & $1845+$ \\
\hline $\begin{array}{l}{[9]} \\
\text { Binh et } \\
a l, 2001\end{array}$ & $\begin{array}{l}17 \text { healthy Vietnamese volunteers; subjects } \\
\text { randomized into two groups, both receiving: } \\
120 \text { mg IV AS over } 2 \text { min }\end{array}$ & $\begin{array}{l}1507 ; \\
1678+\end{array}$ & $9 ; 16$ & & & $53 ; 47$ & \\
\hline $\begin{array}{l}\text { [10] } \\
\text { llett et al, } \\
2002\end{array}$ & $\begin{array}{l}23 \text { Vietnamese adults with uncomplicated } \\
\text { falciparum malaria; subjects randomized into two } \\
\text { groups, both receiving: } \\
120 \text { mg IV AS over } 2 \text { min }\end{array}$ & $\begin{array}{l}2758 \\
2730 \dagger^{a}\end{array}$ & $7 ; 9^{a}$ & $0.64 ; 0.48$ & $0.8 ; 0.55$ & $59 ; 50$ & $2872 ; 3298+$ \\
\hline $\begin{array}{l}{[11]} \\
\text { Li et al, } \\
2009\end{array}$ & $\begin{array}{l}30 \text { healthy volunteers } \\
0.5,1,2,4 \text {, or } 8 \mathrm{mg} / \mathrm{kg} \text { IV AS over } 2 \mathrm{~min}\end{array}$ & $\begin{array}{l}428 ; 802 ; \\
1286 ; 3148 ; \\
4744\end{array}$ & $\begin{array}{l}9.6 ; 15 ; 9.6 ; \\
7.2 ; 24 t\end{array}$ & $\begin{array}{l}1.3 ; 0.98 \\
1.1 ; 0.86 \\
0.82+\end{array}$ & $\begin{array}{l}\text { Vss: } 1.734 ; \\
2.201 ; 1.860 ; \\
1.701 ; 2.403+\end{array}$ & $\begin{array}{l}57.6 ; 92.4 ; \\
69.0 ; 82.2 ; \\
128.4+\end{array}$ & $\begin{array}{l}385 ; 1082 ; \\
1850 ; 4886 ; \\
10410\end{array}$ \\
\hline $\begin{array}{l}{[12]} \\
\text { Nealon } \\
\text { et al, } \\
2002\end{array}$ & $\begin{array}{l}28 \text { paediatric Gabonese patients with severe } \\
\text { malaria randomized into two groups } \\
\text { Group 1: } 2.4 \mathrm{mg} / \mathrm{kg} \text { IV AS } \\
\text { Group 2: } 1.2 \mathrm{mg} / \mathrm{kg} \text { IV AS }\end{array}$ & $\begin{array}{l}\text { Group 1: } \\
3011 t^{a} \\
\text { Group 2: } \\
1584 t^{a}\end{array}$ & $\begin{array}{l}\text { Group 1: } \\
0.5^{\mathrm{a}} \\
\text { Group 2: } \\
1.4^{\mathrm{a}}\end{array}$ & $\begin{array}{l}\text { Group 1: } \\
2.16 t^{\mathrm{a}} \\
\text { Group 2: } \\
1.08 t^{\mathrm{a}}\end{array}$ & $\begin{array}{l}\text { Group } 1 \\
\text { Vss: } 0.75^{a} \\
\text { Group } 2 \\
\text { Vss: } 0.77^{\text {a }}\end{array}$ & $\begin{array}{l}\text { Group 1: } \\
20.7^{\mathrm{a}} \\
\text { Group 2: } \\
32.0^{\mathrm{a}}\end{array}$ & $\begin{array}{l}\text { Group 1: } \\
923 t^{\mathrm{a}} \\
\text { Group 2: } \\
737 \dagger^{\mathrm{a}}\end{array}$ \\
\hline $\begin{array}{l}\text { [13] } \\
\text { Newton } \\
\text { et al, } \\
2006\end{array}$ & $\begin{array}{l}17 \text { adults with severe falciparum malaria in Thailand } \\
2.4 \mathrm{mg} / \mathrm{kg} \text { IV AS over } 2 \mathrm{~min} \\
{[2.1(1.4-2.8 \mathrm{mg} / \mathrm{kg})]}\end{array}$ & $605 t^{a}$ & $\begin{array}{l}\text { Tmax } \\
\text { reached by } \\
15 \text { min }\end{array}$ & $5.6^{\mathrm{a}}$ & Vss: $1.9^{\mathrm{a}}$ & $20.4 t^{a}$ & $418 t^{a}$ \\
\hline $\begin{array}{l}8] \\
\text { Batty. } \\
\text { Thu et al, } \\
1998\end{array}$ & $\begin{array}{l}26 \text { adult uncomplicated falciparum malaria patients } \\
\text { in Vietnam } \\
120 \text { mg IV AS over } 2 \text { min }\end{array}$ & $2648 t^{a}$ & $9.0^{a}$ & $0.75^{a}$ & 0.76 & 40.2 & $2377+$ \\
\hline $\begin{array}{l}{[14]} \\
\text { Davis et } \\
\text { al, } 2001\end{array}$ & $\begin{array}{l}30 \text { parasitaemic adults with falciparum malaria } \\
\text { Group 1: } 12 \text { with complications } \\
\text { Group 2: } 8 \text { without complications } \\
\text { Group 1 \& 2: } \\
120 \text { mg IV AS over } 2 \text { min } \\
\text { Group 3: } 10 \text { with moderately severe complications } \\
\text { (240 mg IV AS infused over } 4 \text { hours; samples taken } \\
\text { after infusion discontinued) }\end{array}$ & $\begin{array}{l}\text { Group 1: } \\
2417+ \\
\text { Group 2: } \\
2531+ \\
\text { Group 3: } \\
910+\end{array}$ & $\begin{array}{l}\text { Group 1: } \\
10.4 \\
\text { Group 2: } \\
9.9 \\
\text { Group 3: } \\
240 \text { (end } \\
\text { of infusion) }\end{array}$ & $\begin{array}{l}\text { Group 1: } \\
1.09 \\
\text { Group 2: } \\
0.73 \\
\text { Group 3: } \\
0.73\end{array}$ & $\begin{array}{l}\text { Group 1: } \\
0.77 \\
\text { Group 2: } \\
\text { 1.01 } \\
\text { Group 3: } \\
0.78\end{array}$ & $\begin{array}{l}\text { Group 1: } \\
40.0 \\
\text { Group 2: } \\
64.1 \\
\text { Group 3: } \\
46.2\end{array}$ & $\begin{array}{l}\text { Group 1: } \\
2078+ \\
\text { Group 2: } \\
\text { 2559+ } \\
\text { Group 3: } \\
5573+\end{array}$ \\
\hline $\begin{array}{l}\text { Krishna } \\
\text { et al, } \\
2001 \\
{[15]}\end{array}$ & $\begin{array}{l}34 \text { Ghanaian children (8 months - } 7 \text { years) with } \\
\text { moderate falciparum malaria } \\
\text { Group } 1 \text { \& 2: AS rectal suppository, IV AS } 2.4 \text { mg/kg } \\
12 \text { hr later } \\
\text { Group 3: } 2.4 \mathrm{mg} / \mathrm{kg} \text { IV AS, AS rectal suppository } 12 \\
\text { hr later }\end{array}$ & $\begin{array}{l}\text { Groups } 1 \& \\
\text { 2: } \\
\text { 1280t } \\
\text { Group 3: } \\
1592 t^{\mathrm{a}}\end{array}$ & $\begin{array}{l}\text { Groups } 1 \& \\
2: \\
12 \dagger^{a} \\
\text { Group 3: } \\
12+\end{array}$ & $\begin{array}{l}\text { Groups } 1 \& \\
2: \\
1.5^{\mathrm{a}} \\
\text { Group 3: } \\
1.0^{\mathrm{a}}\end{array}$ & $\begin{array}{l}\text { Groups } 1 \& 2: \\
0.6^{\mathrm{a}} \\
\text { Group 3: } \\
0.9^{\mathrm{a}}\end{array}$ & $\begin{array}{l}\text { Groups } 1 \& \\
2: \\
18 t^{a} \\
\text { Group 3: } \\
31.8 t^{a}\end{array}$ & $\begin{array}{l}\text { Groups } 1 \& \\
2: \\
1166 t^{\mathrm{a}} \\
\text { Group 3: } \\
1706 t^{\mathrm{a}}\end{array}$ \\
\hline
\end{tabular}

Values given as mean unless otherwise specified.

tUnits converted to uniform scale a. Median

thalassaemic and healthy non-thalassaemic adults. The reported half-life estimates for normal and thalassaemic subjects were 1.37 and 1.95 hours, respectively, with Tmax for bioassay activity occurring by the first sampling point (15 minutes) [20]. As would be expected given the more extended half-life of DHA, these bioassay results appear somewhat more reflective of the DHA results than the AS results derived from traditional analytical methods.

\section{Intramuscular administration: artesunate and DHA pharmacokinetics}

Tables 3 and 4[10,12,21] summarize the results of the identified studies examining AS and DHA PK following
IM AS administration. Average estimates of the absolute bioavailability of IM AS, as determined by DHA concentrations, were $86.4 \%$ [12] and $88 \%$ [10] in adult and paediatric falciparum malaria patients, respectively. The average time to maximum AS concentrations following IM administration ranged from 7.2 - 12 minutes; average AS half-life ranged from 25.2 - 48.2 minutes. This more extended AS half-life following IM administration of AS presumably indicates that the AS elimination rate was being limited by the rate of absorption from the site of injection. Average estimates for AS apparent clearance and volume of distribution ranged from 2.4 - 3.48 $\mathrm{L} / \mathrm{kg} / \mathrm{hr}$ and $1.09-3.98 \mathrm{~L} / \mathrm{kg}$, respectively. As would be expected given the high bioavailability of IM AS, these 
Table 3 Summary of AS pharmacokinetic results following IM AS administration

\begin{tabular}{|c|c|c|c|c|c|c|c|}
\hline Ref. & Subjects \& Regimen & $\begin{array}{l}\text { Cmax } \\
(\mathrm{ng} / \mathrm{mL})\end{array}$ & $\begin{array}{l}\text { Tmax } \\
(\min )\end{array}$ & $\begin{array}{l}\text { Clearance } \\
(\mathrm{L} / \mathrm{kg} / \mathrm{hr})\end{array}$ & $\begin{array}{l}\text { Volume } \\
(\mathrm{L} / \mathrm{kg})\end{array}$ & $\begin{array}{l}\text { Half-life } \\
\text { (min) }\end{array}$ & $\begin{array}{l}\text { AUC } \\
\text { (ng*hr/mL) }\end{array}$ \\
\hline $\begin{array}{l}10] \\
\text { llett et al, } \\
2002 \\
\end{array}$ & $\begin{array}{l}11 \text { Vietnamese adults with uncomplicated falciparum } \\
\text { malaria } \\
120 \text { mg AS administered by intramuscular injection }\end{array}$ & $884 t^{a}$ & $12^{a}$ & 2.9 & 2.6 & 41 & $999+$ \\
\hline $\begin{array}{l}{[21]} \\
\text { Hien et al, } \\
2004\end{array}$ & $\begin{array}{l}9 \text { Vietnamese adults with severe falciparum malaria } \\
2.4 \mathrm{mg} / \mathrm{kg} \text { AS administered by intramuscular injection }\end{array}$ & $2195 t^{a}$ & & $2.84^{a}$ & $1.09^{\mathrm{a}}$ & $30^{a}$ & $856 t^{a}$ \\
\hline $\begin{array}{l}{[12]} \\
\text { Nealon et al, } \\
2002\end{array}$ & $\begin{array}{l}28 \text { paediatric Gabonese patients with severe malaria } \\
\text { randomized into two groups } \\
\text { Group 1: } 2.4 \mathrm{mg} / \mathrm{kg} \text { IV AS, followed by } 1.2 \mathrm{mg} / \mathrm{kg} \text { IM AS } \\
12 \text { hours later } \\
\text { Group 2: } 2.4 \mathrm{mg} / \mathrm{kg} \text { IM AS, followed by } 1.2 \mathrm{mg} / \mathrm{kg} \text { IV AS } \\
12 \text { hours later }\end{array}$ & $\begin{array}{l}\text { Group 1: } \\
615 t^{\mathrm{a}} \\
\text { Group 2: } \\
661 t^{\mathrm{a}}\end{array}$ & $\begin{array}{l}\text { Group } \\
1: \\
7.2^{\mathrm{a}} \\
\text { Group } \\
2: \\
8.0^{\mathrm{a}}\end{array}$ & $\begin{array}{l}\text { Group 1: } \\
2.4 t^{\mathrm{a}} \\
\text { Group 2: } \\
3.48+^{\mathrm{a}}\end{array}$ & $\begin{array}{l}\text { Group } 1 \mathrm{Vss} / \\
\text { F: } 2.07^{\mathrm{a}} \\
\text { Group } 2 \mathrm{Vss} / \\
\text { F: } 3.98^{\mathrm{a}}\end{array}$ & $\begin{array}{l}\text { Group 1: } \\
25.2^{\mathrm{a}} \\
\text { Group 2: } \\
48.2^{\mathrm{a}}\end{array}$ & $\begin{array}{l}\text { Group 1: } \\
535 t^{\mathrm{a}} \\
\text { Group 2: } \\
544 t^{\mathrm{a}}\end{array}$ \\
\hline
\end{tabular}

Values given as mean unless otherwise specified.

tUnits converted to uniform scale a. Median

estimates for apparent clearance are not strikingly higher than those obtained following IV AS administration.

Maximum DHA concentrations following IM AS administration occurred, on average, within the first 45 minutes post-dose. Average estimates for DHA half-life, apparent clearance, and apparent volume of distribution following IM AS ranged from 31.9 - 64 minutes, 0.73 $2.16 \mathrm{~L} / \mathrm{kg} / \mathrm{hr}$, and $1.1-1.7 \mathrm{~L} / \mathrm{kg}$, respectively; all of these values are quite similar to those obtained following IV AS administration.

\section{Oral administration: artesunate pharmacokinetics}

Based upon complete metabolism to DHA, AS displays high oral bioavailability when assessed by exposure to its active metabolite DHA. Following intravenous and oral AS, the oral bioavailability of DHA was determined to be $82 \%$ in healthy adults [9], $85 \%$ in adults with uncomplicated falciparum malaria [8], and $80 \%$ in adults with vivax malaria [7]. It should be noted, however, that these bioavailability results may reflect both the absorption of AS, with subsequent conversion to DHA through first-pass or systemic metabolism, as well as direct absorption of DHA following its formation in the gut through acid-dependent chemical hydrolysis [22]. Although physiologically plausible, the extent of such chemical hydrolysis has not been well quantified. Following oral administration, AS concentrations are detectable early, often within 15 minutes post-dose. Peak AS concentrations also occur early, with AS Tmax typically being detected within the first hour post-dose (Table 5; [6,23-37]). These findings suggest that AS is absorbed quickly and without appreciable lag.

AS half-life estimates available in the literature are summarized in Table 6 ([28-31,35-37]), with average AS half-life reported for any cohort in the identified studies ranging from $0.36-1.2$ hours. All studies in which AS half-life was determined cite AS half-life values between 25 and 40 minutes for at least one study cohort. There are few published estimates of AS apparent clearance

Table 4 Summary of DHA pharmacokinetic results following IM AS administration

\begin{tabular}{|c|c|c|c|c|c|c|c|}
\hline Ref. & Subjects \& Regimen & $\begin{array}{l}\text { Cmax } \\
(\mathrm{ng} / \mathrm{mL})\end{array}$ & $\begin{array}{l}\text { Tmax } \\
\text { (min) }\end{array}$ & $\begin{array}{l}\text { Clearance } \\
\text { (L/kg/hr) }\end{array}$ & $\begin{array}{l}\text { Volume } \\
(\mathrm{L} / \mathrm{kg})\end{array}$ & $\begin{array}{l}\text { Half-life } \\
\text { (min) }\end{array}$ & $\begin{array}{l}\text { AUC } \\
\left(\mathrm{ng}^{*} \mathrm{hr} / \mathrm{mL}\right)\end{array}$ \\
\hline $\begin{array}{l}\text { [10] } \\
\text { llett et al, } \\
2002\end{array}$ & $\begin{array}{l}11 \text { Vietnamese adults with uncomplicated falciparum } \\
\text { malaria } \\
120 \text { mg AS administered by intramuscular injection }\end{array}$ & $1166 t^{a}$ & $45^{a}$ & 0.73 & 1.1 & 64 & $\begin{array}{l}2474+ \\
F: 88 \%\end{array}$ \\
\hline $\begin{array}{l}{[21]} \\
\text { Hien et al, } \\
2004\end{array}$ & $\begin{array}{l}9 \text { Vietnamese adults with severe falciparum malaria } \\
2.4 \mathrm{mg} / \mathrm{kg} \mathrm{AS} \text { administered by intramuscular injection }\end{array}$ & $870 t^{a}$ & $35^{a}$ & $1.18^{a}$ & $1.79^{a}$ & $52.7^{a}$ & $1496 t^{a}$ \\
\hline $\begin{array}{l}\text { [12] } \\
\text { Nealon et } \\
\text { al, } 2002\end{array}$ & $\begin{array}{l}28 \text { paediatric Gabonese patients with severe malaria } \\
\text { randomized into two groups } \\
\text { Group } 1: 2.4 \mathrm{mg} / \mathrm{kg} \text { IV AS, followed by } 1.2 \mathrm{mg} / \mathrm{kg} \text { IM } \\
\text { AS } 12 \text { hours later } \\
\text { Group } 2: 2.4 \mathrm{mg} / \mathrm{kg} \mathrm{IM} \mathrm{AS,} \mathrm{followed} \mathrm{by} 1.2 \mathrm{mg} / \mathrm{kg} \text { IV } \\
\text { AS } 12 \text { hours later }\end{array}$ & $\begin{array}{l}\text { Group 1: } \\
341^{a} \\
\text { Group 2: } \\
626^{a}\end{array}$ & $\begin{array}{l}\text { Group } \\
1: \\
25.9^{a} \\
\text { Group } \\
2: \\
40.5^{\text {a }}\end{array}$ & $\begin{array}{l}\text { Group 1: } \\
2.16 t^{a} \\
\text { Group 2: } \\
1.5 t^{a}\end{array}$ & $\begin{array}{l}\text { Group 1: } \\
\text { Vc/F: } 1.2^{a} \\
\text { Vss/F: } \\
1.32^{a} \\
\text { Group 2: } \\
\text { Vc/F: } 1.2^{a} \\
\text { Vss/F: } \\
1.28^{a}\end{array}$ & $\begin{array}{l}\text { Group 1: } \\
31.9^{\mathrm{a}} \\
\text { Group 2: } \\
40.2^{\mathrm{a}}\end{array}$ & $\begin{array}{l}\text { Group 1: } \\
396 t^{\mathrm{a}} \\
\text { Group 2: } \\
1123 \dagger^{\mathrm{a}} \\
\text { Combined group } \\
\text { F: } 86.37 \%^{\mathrm{a}}\end{array}$ \\
\hline
\end{tabular}

Values given as mean unless otherwise specified.

tUnits converted to uniform scale a. Median 
Table 5 Artesunate Tmax values obtained following oral artesunate administration

\begin{tabular}{|c|c|c|c|}
\hline Ref. & Subjects & Regimen & Tmax (hours) \\
\hline [23] & 15 healthy Cambodian male adults & $4 \mathrm{mg} / \mathrm{kg}$ AS once with mefloquine & $0.75^{\mathrm{a}}$ \\
\hline [6] & 8 healthy adults in Australia & $150 \mathrm{mg}$ AS once & $0.65(n=6)^{a}$ \\
\hline [24] & 20 healthy adult males in Australia & $\begin{array}{l}200 \mathrm{mg} / \text { day } \times 3 \text { days alone (Period 1); repeated } \\
\text { with mefloquine after washout (Period 2) }\end{array}$ & $\begin{array}{l}\text { Period 1/Day 1: } \\
0.6^{\mathrm{b}} \\
\text { Period 1/Day 3: } \\
0.6^{\mathrm{b}} \\
\text { Period 2/Day1: } \\
0.5^{\mathrm{b}} \\
\text { Period 2/Day 3: } \\
0.6^{\mathrm{b}}\end{array}$ \\
\hline [25] & 6 healthy adults in Geneva & $200 \mathrm{mg}$ AS once & $\begin{array}{l}0.25 \text { ( } 5 / 6 \text { subjects }) \\
0.5 \text { ( } 1 / 6 \text { subjects) } \\
\end{array}$ \\
\hline [26] & 23 healthy Malaysian adults & $\begin{array}{l}200 \mathrm{mg} \text { AS once with amodiaquine as fixed or } \\
\text { non-fixed product }\end{array}$ & $\begin{array}{l}\text { Fixed:0.26 } \\
\text { Non-fixed: } 0.53\end{array}$ \\
\hline$[27]$ & 13 healthy adults in Africa & $\begin{array}{l}\text { Mean dose: } 4.26 \mathrm{mg} / \mathrm{kg} \text { with (ACT) or without } \\
\text { (AS only) amodiaquine as single dose }\end{array}$ & $\begin{array}{l}\text { AS only: } 0.62 \\
\text { ACT: } 0.86\end{array}$ \\
\hline [28] & 8 healthy male Thai adults & 300 mg AS (Guilin or Arenco formulation) & $\begin{array}{l}\text { Guilin: } 0.25^{\mathrm{a}} \\
\text { Arenco: } 0.31^{\mathrm{a}}\end{array}$ \\
\hline$[29]$ & 10 healthy male Vietnamese adults & $200 \mathrm{mg}$ AS once daily $\times 5$ days & $\begin{array}{l}\text { Day 1: } 0.8^{\mathrm{a}} \\
\text { Day 5: } 0.8^{\mathrm{a}}\end{array}$ \\
\hline [30] & 12 healthy male Malaysian adults & $200 \mathrm{mg}$ AS once & $0.66 \pm 0.34$ \\
\hline$[31]$ & 11 male Thai adults with uncomplicated falciparum malaria & $\begin{array}{l}200 \mathrm{mg} \text { AS once, followed by } 100 \mathrm{mg} 12 \text { hours } \\
\text { later, then } 100 \mathrm{mg} \text { once daily for another } 4 \text { days }\end{array}$ & $\begin{array}{l}\text { Acute: } 0.5^{\mathrm{a}} \\
\text { Convalescence: } 1.0^{\mathrm{a}}\end{array}$ \\
\hline$[32]$ & 43 adults with uncomplicated falciparum malaria in Thailand & $\begin{array}{l}\text { AS+mefloquine as fixed ( } 200 \mathrm{mg} \mathrm{AS}) \text { or } \\
\text { nonfixed ( } 4 \mathrm{mg} / \mathrm{kg} \mathrm{AS} \text { ) combination }\end{array}$ & $\begin{array}{l}\text { Fixed:0.833 }(\mathrm{n}= \\
19) \\
\text { Nonfixed:0.925 ( } \\
=23 \text { ) }\end{array}$ \\
\hline [33] & $\begin{array}{l}13 \text { male and female adult patients in the DRC with acute } \\
\text { uncomplicated falciparum malaria }\end{array}$ & $\begin{array}{l}200 \mathrm{mg} \text { AS once daily } \times 3 \text { days with } \\
\text { amodiaquine }\end{array}$ & $1.4(n=10)$ \\
\hline$[34]$ & $\begin{array}{l}86 \text { acute uncomplicated falciparum malaria patients from Malawi and } \\
\text { Gambia }\end{array}$ & $\begin{array}{l}\text { 1, 2, or } 4 \mathrm{mg} / \mathrm{kg} \text { AS with chlorproguanil and } \\
\text { dapsone once daily } \times 3 \text { days }\end{array}$ & $\begin{array}{l}1 \mathrm{mg} / \mathrm{kg}: 1.08^{\mathrm{a}} \\
2 \mathrm{mg} / \mathrm{kg}: 0.55^{\mathrm{a}} \\
4 \mathrm{mg} / \mathrm{kg}: 1.03^{\mathrm{a}}\end{array}$ \\
\hline$[35]$ & $\begin{array}{l}6 \text { male Thai adults with uncomplicated falciparum malaria and } 6 \text { healthy } \\
\text { male adults }\end{array}$ & $100 \mathrm{mg}$ AS once & $\begin{array}{l}\text { Healthy: } 0.71 \\
\text { Patients: not } \\
\text { determined }\end{array}$ \\
\hline [36] & $\begin{array}{l}57 \text { children (2-14 years) with uncomplicated falciparum malaria in } \\
\text { Gabon }\end{array}$ & $\begin{array}{l}\text { AS dose }(\mathrm{mg} / \mathrm{kg} / \text { day) with pyronaridine } \\
\text { Group A:2.1(1.4-2.4) } \\
\text { Group B:3.3(2.4 - 3.9) } \\
\text { Group C:4.8(3.0 - 6.1) } \\
\text { Group D: } 3.8(3.0-4.3)\end{array}$ & $\begin{array}{l}\text { Group A:0.6 } \\
\text { Group B:0.7 } \\
\text { Group C:1.0 } \\
\text { Group D:0.5 }\end{array}$ \\
\hline$[37]$ & $\begin{array}{l}40 \text { children and adults with uncomplicated falciparum malaria in Pailin, } \\
\text { Cambodia and } 40 \text { adults with uncomplicated falciparum malaria in } \\
\text { Wang Pha, Thailand }\end{array}$ & $\begin{array}{l}\text { At each site: } \\
\text { Group 1: AS monotherapy: } 2 \mathrm{mg} / \mathrm{kg} / \text { day } \times 7 \\
\text { days } \\
\text { Group 2: AS } 4 \mathrm{mg} / \mathrm{kg} / \text { day } \times 3 \text { days }+ \\
\text { mefloquine }\end{array}$ & $\begin{array}{l}\text { Thailand: } \\
\text { Group 1: } 0.38^{\mathrm{a}} \\
\text { Group 2: } 0.50^{\mathrm{a}} \\
\text { Cambodia: } \\
\text { Group 1:0.50 } \\
\text { Group 2: } 1.00^{\mathrm{a}}\end{array}$ \\
\hline
\end{tabular}

Values given as mean unless otherwise specified.

a. Median b. Geometric mean

and volume of distribution assessed following oral AS administration. Teja-Isavadharm et al [35] determined mean AS CL/F and V/F to be $20.6 \mathrm{~L} / \mathrm{kg} / \mathrm{hr}$ and $14.8 \mathrm{~L} /$ $\mathrm{kg}$, respectively, in six healthy adult subjects. Karbwang et al [31] determined average AS CL/F to be $19.2 \mathrm{~L} / \mathrm{kg} /$ hr in 11 Thai adults during the acute phase of uncomplicated malaria infection and $9.6 \mathrm{~L} / \mathrm{kg} / \mathrm{hr}$ during the convalescent phase. Median AS V/F was $6.8 \mathrm{~L} / \mathrm{kg}$ during both phases of infection. Finally, in paediatric Gabonese patients with acute falciparum malaria, estimates for average AS CL/F and V/F were $25-30 \mathrm{~L} / \mathrm{kg} / \mathrm{hr}$ and 25 - $41 \mathrm{~L} / \mathrm{kg}$, respectively [36].

Given that AS is a high extraction ratio drug, the substantial difference in magnitude of these AS apparent clearance and volume estimates for oral administration, as compared to IV or IM administration, most likely 
Table 6 Artesunate half-life values following oral artesunate administration

\begin{tabular}{|c|c|c|c|}
\hline Ref. & Subjects & Artesunate regimen & $\begin{array}{l}\begin{array}{l}\text { Artesunate } \\
\text { half-life } \\
\text { (hours) }\end{array} \\
\end{array}$ \\
\hline$[29]$ & 10 healthy male Vietnamese adults & $200 \mathrm{mg}$ once daily $\times 5$ days & $\begin{array}{l}\text { Day 1: } 0.43^{\mathrm{a}} \\
\text { Day 5: } 0.50^{\mathrm{a}}\end{array}$ \\
\hline$[30]$ & 12 healthy male Malaysian adults & $200 \mathrm{mg}$ single dose & 0.49 \\
\hline [28] & 8 healthy male Thai adults & 300 mg AS (Guilin or Arenco formulation) & $\begin{array}{l}\text { Guilin: } 0.53 \\
\text { Arenco:0.57 }\end{array}$ \\
\hline [26] & 23 healthy Malaysian adults & $\begin{array}{l}200 \mathrm{mg} \mathrm{AS}+\text { amodiaquine as fixed or non- } \\
\text { fixed product }\end{array}$ & $\begin{array}{l}\text { Fixed:0.63 } \\
\text { Non-fixed:0.76 }\end{array}$ \\
\hline$[34]$ & $\begin{array}{l}86 \text { acute uncomplicated falciparum malaria patients from Malawi and } \\
\text { Gambia }\end{array}$ & $\begin{array}{l}\text { 1, 2, or } 4 \mathrm{mg} / \mathrm{kg} \mathrm{AS}+\text { chlorproguanil and } \\
\text { dapsone once daily } \times 3 \text { days }\end{array}$ & $\begin{array}{l}1 \mathrm{mg} / \mathrm{kg}: \\
0.515^{\mathrm{b}} \\
2 \mathrm{mg} / \mathrm{kg}: \\
0.478^{\mathrm{b}} \\
4 \mathrm{mg} / \mathrm{kg}: \\
0.467^{\mathrm{b}}\end{array}$ \\
\hline$[31]$ & 11 male Thai adults with uncomplicated falciparum malaria & $\begin{array}{l}200 \mathrm{mg} \text { AS once, followed by } 100 \mathrm{mg} 12 \\
\text { hours later, then } 100 \mathrm{mg} \text { once daily } \times 4 \text { days }\end{array}$ & $\begin{array}{l}\text { Acute: } 0.36^{\mathrm{a}} \\
\text { Convalescence: } \\
0.54^{\mathrm{a}}\end{array}$ \\
\hline [35] & $\begin{array}{l}6 \text { male Thai adults with uncomplicated falciparum malaria and } 6 \text { healthy } \\
\text { male adults }\end{array}$ & $100 \mathrm{mg}$ AS once & $\begin{array}{l}\text { Healthy: } 0.41 \\
\text { Patients: not } \\
\text { determined }\end{array}$ \\
\hline [36] & 57 children (2-14 years) with uncomplicated falciparum malaria in Gabon & $\begin{array}{l}\text { AS dose }(\mathrm{mg} / \mathrm{kg}) \text { with pyronaridine } \\
\text { Group A:2.1(1.4-2.4) } \\
\text { Group B:3.3(2.4- 3.9) } \\
\text { Group C:4.8(3.0 - 6.1) } \\
\text { Group D: } 3.8(3.0-4.3)\end{array}$ & $\begin{array}{l}\text { Group A: } 0.8 \text { ( } \mathrm{n} \\
=12) \\
\text { Group B:1.1 ( } \\
=12) \\
\text { Group C:0.5 (n } \\
=10) \\
\text { Group D:1.2 (n } \\
=13 \text { ) }\end{array}$ \\
\hline$[37]$ & $\begin{array}{l}40 \text { children and adults with uncomplicated falciparum malaria in Pailin, } \\
\text { Cambodia and } 40 \text { adults with uncomplicated falciparum malaria in Wang } \\
\text { Pha, Thailand }\end{array}$ & $\begin{array}{l}\text { At each site: } \\
\text { Group 1: AS monotherapy: } 2 \mathrm{mg} / \mathrm{kg} / \text { day } \times 7 \\
\text { days } \\
\text { Group 2: AS } 4 \mathrm{mg} / \mathrm{kg} / \text { day } \times 3 \text { days }+ \\
\text { mefloquine }\end{array}$ & $\begin{array}{l}\text { Thailand: } \\
\text { Group 1: } 0.37^{\mathrm{a}} \\
\text { Group 2: } 0.58^{\mathrm{a}} \\
\text { Cambodia: } \\
\text { Group 1: } 0.29^{\mathrm{a}} \\
\text { Group 2: } 0.29^{\mathrm{a}}\end{array}$ \\
\hline
\end{tabular}

Value given as mean unless otherwise specified.

a. Median b. Geometric mean

reflects the low bioavailability of AS due to the extensive conversion of AS to DHA during first-pass metabolism.

\section{Oral administration: DHA pharmacokinetics}

For studies defining both AS and DHA PK following oral AS administration, DHA Cmax exceeds AS Cmax, and DHA AUC exceeds AS AUC. Literature results exemplifying this relationship between AS and DHA pharmacokinetic exposure are summarized in Table 7 [6,26-30,32,34,35,37-39]. In many of these studies, DHA AUC exceeds AS AUC by more than 10-fold, when considered on either a $\mathrm{nmol} * \mathrm{hr} / \mathrm{mL}$ or $\mathrm{ng}^{*} \mathrm{hr} / \mathrm{mL}$ basis. It is in part due to this disparity in exposure that AS is often considered essentially a pro-drug for DHA following oral AS administration. The maximum concentration for DHA typically occurs within two hours post-dose. DHA is eliminated more slowly than AS following oral AS administration. DHA half-life was determined to be longer than AS half-life for all studies in which both parameters were assessed. For the studies in which DHA half-life was estimable (Table 8; [7-9,23-32,34-43]), the average DHA half-life ranged from 0.49 hours to 3.08 hours, with almost half of the half-life estimates being less than one hour and most of the remaining estimates being between 1-2 hours. Overall, most of the half-life estimates fell between 0.5 - 1.5 hours.

DHA CL/F and V/F estimates obtained following oral AS administration are limited. Teja-Isavadharm et al [35] determined DHA CL/F to be $3.35 \mathrm{~L} / \mathrm{kg} / \mathrm{hr}$ in six healthy adults and $1.01 \mathrm{~L} / \mathrm{kg} / \mathrm{hr}$ in six parasitaemic adults. DHA apparent volume of distribution values were $4.14 \mathrm{~L} / \mathrm{kg}$ and $1.55 \mathrm{~L} / \mathrm{kg}$ in healthy subjects and malaria patients, respectively. In Gabonese children with malaria, average DHA CL/F and V/F averaged $2.3-2.7$ $\mathrm{L} / \mathrm{kg} / \mathrm{hr}$ and $1.6-4.2 \mathrm{~L} / \mathrm{kg}$, respectively [36]. Orrell et al [27], Davis et al [24], and Zhang et al [41] computed DHA CL/F, but did not provide values adjusted for body weight. Adjusting CL/F using the average body 
Table 7 Artesunate and DHA AUC and Cmax values following oral artesunate administration

\begin{tabular}{|c|c|c|c|c|}
\hline Ref. & Subjects & Oral AS regimen & $\begin{array}{l}\text { AUC } \\
\left(\mathrm{ng} \mathrm{g}^{*} \mathrm{hr} / \mathrm{mL}\right)\end{array}$ & $\mathrm{Cmax}(\mathrm{ng} / \mathrm{mL})$ \\
\hline \multirow[t]{2}{*}{$\overline{[29]}$} & 10 healthy male Vietnamese adults & $200 \mathrm{mg}$ once daily $\times 5$ days & $\begin{array}{l}\text { AS }^{\text {a }} \\
\text { Day 1: } 67 \\
\text { Day 5: } 60\end{array}$ & $\begin{array}{l}\mathrm{AS}^{a} \\
\text { Day 1:67 } \\
\text { Day 5: } 58\end{array}$ \\
\hline & & & $\begin{array}{l}\text { DHA }^{\text {a }} \\
\text { Day 1:1158 } \\
\text { Day 5:1300 } \\
\end{array}$ & $\begin{array}{l}\text { DHA } \\
\text { Pooled:654 }\end{array}$ \\
\hline \multirow[t]{2}{*}{ [30] } & 12 healthy male Malaysian adults & $200 \mathrm{mg}$ AS once & $\begin{array}{l}\text { AS } \\
119 \\
\end{array}$ & $\begin{array}{l}\text { AS } \\
256.3 \\
\end{array}$ \\
\hline & & & $\begin{array}{l}\text { DHA } \\
\text { AUC }_{0-\mathrm{t}: 1331}\end{array}$ & $\begin{array}{l}\text { DHA } \\
873.7\end{array}$ \\
\hline \multirow[t]{2}{*}[27]{} & 13 healthy adults in Africa & $\begin{array}{l}\text { Mean dose: } 4.26 \mathrm{mg} / \mathrm{kg} \text { with (ACT) or without } \\
\text { (AS only) amodiaquine single dose }\end{array}$ & $\begin{array}{l}\text { AS } \\
\text { AS only: } 206.4 \\
\text { ACT: } 183.3\end{array}$ & $\begin{array}{l}\text { AS } \\
\text { AS only: } 231.8 \\
\text { ACT: } 141.6\end{array}$ \\
\hline & & & $\begin{array}{l}\text { DHA } \\
\text { AS only: } \\
2044.4 \\
\text { ACT: } 1410.5\end{array}$ & $\begin{array}{l}\text { DHA } \\
\text { AS only: } 844.5 \\
\text { ACT: } 446.2\end{array}$ \\
\hline \multirow[t]{2}{*}[28]{} & 8 healthy male Thai adults & $\begin{array}{l}300 \mathrm{mg} \text { AS } \\
\text { (Guilin and Arenco formulations) }\end{array}$ & $\begin{array}{l}\text { AS } \\
\text { Guilin: } 406 \\
\text { Arenco: } 190.8\end{array}$ & $\begin{array}{l}\text { AS } \\
\text { Guilin: } 397 \\
\text { Arenco: } 194 \\
\end{array}$ \\
\hline & & & $\begin{array}{l}\text { DHA } \\
\text { Guilin: } 1630 \\
\text { Arenco: } 2600\end{array}$ & $\begin{array}{l}\text { DHA } \\
\text { Guilin: } 500 \\
\text { Arenco:928 }\end{array}$ \\
\hline \multirow[t]{2}{*}{$\overline{[6]}$} & 8 healthy adults in Australia & $150 \mathrm{mg}$ once & $\begin{array}{l}\text { AS } \\
\text { AUC } C_{0-6} \text { hr:154 } \\
(n=6)\end{array}$ & $\begin{array}{l}\mathrm{AS}^{\mathrm{a}} \\
111(\mathrm{n}=6)\end{array}$ \\
\hline & & & $\begin{array}{l}\text { DHA } \\
\text { AUC }_{0-6 \text { hr: }}: 824\end{array}$ & $\begin{array}{l}\mathrm{DHA}^{\mathrm{a}} \\
546\end{array}$ \\
\hline \multirow[t]{2}{*}[26]{} & 23 healthy Malaysian adults & $\begin{array}{l}200 \mathrm{mg} \text { once with amodiaquine as fixed or } \\
\text { non-fixed product }\end{array}$ & $\begin{array}{l}\text { ASt } \\
\text { Fixed: } 391.1 \\
\text { Non-fixed: } \\
213.2\end{array}$ & $\begin{array}{l}\text { ASt } \\
\text { Fixed: } 333 \\
\text { Non-fixed: } 444\end{array}$ \\
\hline & & & $\begin{array}{l}\text { DHAt } \\
\text { Fixed: } 1468.9 \\
\text { Non-fixed: } \\
1656.0\end{array}$ & $\begin{array}{l}\text { DHAt } \\
\text { Fixed: } 609.8 \\
\text { Non-fixed:874.5 }\end{array}$ \\
\hline \multirow[t]{2}{*}[34]{} & $\begin{array}{l}86 \text { acute uncomplicated falciparum malaria patients from } \\
\text { Malawi and Gambia }\end{array}$ & $\begin{array}{l}\text { 1, 2, or } 4 \mathrm{mg} / \mathrm{kg} \text { AS with chlorproguanil and } \\
\text { dapsone once daily } \times 3 \text { days }\end{array}$ & $\begin{array}{l}\mathrm{AS}^{\mathrm{b}} \\
1 \mathrm{mg} / \mathrm{kg}: 64.6 \\
(\mathrm{n}=16) \\
2 \mathrm{mg} / \mathrm{kg}: 151 \\
(\mathrm{n}=19) \\
4 \mathrm{mg} / \mathrm{kg}: 400 \\
(n=23)\end{array}$ & $\begin{array}{l}\mathrm{AS}^{\mathrm{b}} \\
1 \mathrm{mg} / \mathrm{kg}: 48.9 \\
2 \mathrm{mg} / \mathrm{kg}: 106 \\
4 \mathrm{mg} / \mathrm{kg}: 224\end{array}$ \\
\hline & & & $\begin{array}{l}\mathrm{DHA}^{\mathrm{b}} \\
1 \mathrm{mg} / \mathrm{kg}: 538 \\
(\mathrm{n}=24) \\
2 \mathrm{mg} / \mathrm{kg}: 1445 \\
(\mathrm{n}=29) \\
4 \mathrm{mg} / \mathrm{kg}: 383 \\
(\mathrm{n}=23)\end{array}$ & $\begin{array}{l}\mathrm{DHA}^{\mathrm{b}} \\
1 \mathrm{mg} / \mathrm{kg}: 228 \\
2 \mathrm{mg} / \mathrm{kg}: 581 \\
4 \mathrm{mg} / \mathrm{kg}: 1414\end{array}$ \\
\hline \multirow[t]{2}{*}{ [39] } & $\begin{array}{l}21 \text { children ( } 5 \text { - } 13 \text { years) with uncomplicated malaria in } \\
\text { Uganda }\end{array}$ & $\begin{array}{l}4 \mathrm{mg} / \mathrm{kg} \text { once daily with amodiaquine } \times 3 \\
\text { days }\end{array}$ & $\begin{array}{l}\text { AS } \\
113 \text { (data } \\
\text { pooled from } \\
\text { all subjects) }\end{array}$ & $\begin{array}{l}\text { AS } 51 \text { (data } \\
\text { pooled from all } \\
\text { subjects) }\end{array}$ \\
\hline & & & $\begin{array}{l}\mathrm{DHA}^{\mathrm{a}} \\
1404\end{array}$ & $\begin{array}{l}\mathrm{DHA}^{\mathrm{a}} \\
473\end{array}$ \\
\hline$\overline{[32]}$ & 43 adults with uncomplicated falciparum malaria in Thailand & $\begin{array}{l}200 \mathrm{mg} / \text { day for fixed dose AS-mefloquine } \\
\text { tablet }(\mathrm{n}=20 \text { ) or } 4 \mathrm{mg} / \mathrm{kg} / \text { day as nonfixed ( } \mathrm{n} \\
=23 \text { ) AS-mefloquine }\end{array}$ & $\begin{array}{l}\text { AS } \\
\text { AUC } C_{0-t}: \\
\text { Fixed:310 } \\
(n=19) \\
\text { Nonfixed: } 419 \\
(n=21)\end{array}$ & $\begin{array}{l}\text { AS } \\
\text { Fixed:255 } \\
(n=19) \\
\text { Nonfixed:451 } \\
(n=23)\end{array}$ \\
\hline
\end{tabular}


Table 7 Artesunate and DHA AUC and Cmax values following oral artesunate administration (Continued)

\begin{tabular}{|c|c|c|c|c|}
\hline & & & $\begin{array}{l}\text { DHA } \\
\text { AUC } \text { O-t: } \\
\text { Fixed:3027 } \\
\text { Nonfixed: } \\
3633\end{array}$ & $\begin{array}{l}\text { DHA } \\
\text { Fixed:1234 } \\
\text { Nonfixed:2043 }\end{array}$ \\
\hline \multirow[t]{2}{*}{ [35] } & $\begin{array}{l}6 \text { male Thai adults with uncomplicated falciparum malaria } \\
\text { and } 6 \text { healthy male adults }\end{array}$ & $100 \mathrm{mg}$ AS once & $\begin{array}{l}\text { AS } \\
\text { AUC } 0-12 \text { hr } \\
\text { Healthy: } 97 \\
\text { Patients: not } \\
\text { determined }\end{array}$ & $\begin{array}{l}\text { AS } \\
\text { Healthy: } 114 \\
\text { Patients: not } \\
\text { determined }\end{array}$ \\
\hline & & & $\begin{array}{l}\text { DHA } \\
\text { AUC }_{0-12 \mathrm{hr}} \\
\text { Healthy: } 501 \\
\text { Patients: } 1144\end{array}$ & $\begin{array}{l}\text { DHA } \\
\text { Healthy: } 339 \\
\text { Patients: } 554\end{array}$ \\
\hline \multirow[t]{2}{*}{ [36] } & $\begin{array}{l}57 \text { children ( } 2-14 \text { years) with uncomplicated falciparum } \\
\text { malaria in Gabon }\end{array}$ & $\begin{array}{l}\text { AS dose }(\mathrm{mg} / \mathrm{kg}) \\
\text { Group A:2.1(1.4-2.4) } \\
\text { Group B:3.3(2.4 - 3.9) } \\
\text { Group C:4.8(3.0 - 6.1) } \\
\text { Group D: } 3.8(3.0-4.3)\end{array}$ & $\begin{array}{l}\text { AS } \\
\text { Group A: } 104 \\
\text { (n=12) } \\
\text { Group B: } 154 \\
(n=12) \\
\text { Group C: } 232 \\
(n=10) \\
\text { Group D: } 179 \\
(n=13)\end{array}$ & $\begin{array}{l}\text { AS } \\
\text { Group A: } 93 \\
\text { Group B:154 } \\
\text { Group C: } 287 \\
\text { Group D: } 171\end{array}$ \\
\hline & & Administered with pyronaridine & $\begin{array}{l}\text { DHA } \\
\text { Group A: } 1055 \\
\text { Group B: } 1989 \\
\text { Group C: } 2961 \\
\text { Group D: } 2245\end{array}$ & $\begin{array}{l}\text { DHA } \\
\text { Group A:479 } \\
\text { Group B: } 940 \\
\text { Group C:1186 } \\
\text { Group D:792 }\end{array}$ \\
\hline \multirow[t]{2}{*}[37]{} & $\begin{array}{l}40 \text { children and adults with uncomplicated falciparum } \\
\text { malaria in Pailin, Cambodia and } 40 \text { adults with } \\
\text { uncomplicated falciparum malaria in Wang Pha, Thailand }\end{array}$ & $\begin{array}{l}\text { At each site: } \\
\text { Group 1: AS monotherapy: } 2 \mathrm{mg} / \mathrm{kg} / \text { day } \times 7 \\
\text { days } \\
\text { Group 2: AS } 4 \mathrm{mg} / \mathrm{kg} / \text { day } \times 3 \text { days }+ \\
\text { mefloquine }\end{array}$ & $\begin{array}{l}\text { ASt }^{\mathrm{a}} \\
\text { AUC C-24 hr } \\
\text { Thailand: } \\
\text { Group 1: } 128 \\
\text { Group 2: } 237 \\
\text { Cambodia: } \\
\text { Group 1: } 173 \\
\text { Group 2: } 338\end{array}$ & $\begin{array}{l}\text { ASta }^{a} \\
\text { Thailand: } \\
\text { Group 1: } 171 \\
\text { Group 2: } 200 \\
\text { Cambodia: } \\
\text { Group 1: } 270 \\
\text { Group 2: } 316\end{array}$ \\
\hline & & & $\begin{array}{l}\text { DHAt }^{\mathrm{a}} \\
\text { AUC Co-24 hr } \\
\text { Thailand: } \\
\text { Group 1: } 1308 \\
\text { Group 2: } 2957 \\
\text { Cambodia: } \\
\text { Group 1: } 1382 \\
\text { Group 2: } 4123\end{array}$ & $\begin{array}{l}\text { DHAt }^{a} \\
\text { Thailand: } \\
\text { Group 1: } 859 \\
\text { Group 2: } 1191 \\
\text { Cambodia: } \\
\text { Group 1: } 802 \\
\text { Group 2: } 1590\end{array}$ \\
\hline
\end{tabular}

Values given as mean unless otherwise specified.

tUnits converted to uniform scale a. Median b. Geometric mean

weight in these studies yields apparent clearance estimates of $2.2 \mathrm{~L} / \mathrm{kg} / \mathrm{hr}$ (AS alone) and $2.7 \mathrm{~L} / \mathrm{kg} / \mathrm{hr}$ (with amodiaquine) for Orrell et al [27], $1.4-1.7 \mathrm{~L} / \mathrm{kg} / \mathrm{hr}$ for Davis et al [24], and $1.8 \mathrm{~L} / \mathrm{kg} / \mathrm{hr}$ for Zhang et al [41]. Adjusted DHA V/F values ranged from $1.6-2.6 \mathrm{~L} / \mathrm{kg}$ [24].

\section{Oral administration: bioassay results}

As was previously observed for IV bioassay studies, PK parameters derived from bioassay data obtained following oral AS administration appear to more closely resemble DHA rather than AS parameters. For example, in the four identified studies including bioassay data following oral AS administration, the average Tmax for bioactivity ranged from $0.75-1.7$ hours and average half-life from 0.71 - 1.17 hours [19,44-46].

Oral administration: population pharmacokinetic analyses Four population pharmacokinetic analyses describing AS and/or DHA PK following oral AS administration were identified, including two conducted with data from pregnant women (described under Artesunate and DHA pharmacokinetics in pregnant women, below), as well as analyses conducted using data from healthy volunteers and from paediatric malaria patients. Specifically, Tan et al [47] modelled the PK of AS and DHA simultaneously utilizing extensive sampling data from 91 healthy Korean adults administered oral AS. The data were fit to a 
Table 8 DHA half-life values obtained following artesunate administration

\begin{tabular}{lll}
\hline Ref. & Subjects & Artesunate regimen \\
\hline$[29]$ & 10 healthy male Vietnamese adults & $200 \mathrm{mg}$ once daily $\times 5$ days \\
\hline$[30]$ & 12 healthy male Malaysian adults & $200 \mathrm{mg}$ single dose \\
\hline$[40] \quad 20$ male and female healthy Thai adults & $4 \mathrm{mg} / \mathrm{kg}$ once \\
\hline$[27] \quad 13$ healthy adults in Africa & $\begin{array}{l}\text { Mean dose: } 4.26 \mathrm{mg} / \mathrm{kg} \text { with (ACT) or without (AS } \\
\text { only) amodiaquine single dose }\end{array}$ \\
\hline$[28]$ & 8 healthy male Thai adults & $300 \mathrm{mg}$ AS (Guilin or Arenco formulation) \\
\hline$[41]$ & 10 healthy Vietnamese males & $100 \mathrm{mg}$ AS once \\
\hline$[25]$ & 6 healthy adults in Geneva & $200 \mathrm{mg}$ AS once \\
\hline$[26]$ & 23 healthy Malaysian adults & $200 \mathrm{mg}$ once with amodiaquine as fixed or non- \\
& fixed product \\
\hline$[24]$ & 20 healthy adult males in Australia & $\begin{array}{l}200 \mathrm{mg} / \text { day } \times 3 \text { days alone (Period 1); repeated } \\
\text { with mefloquine after washout (Period 2) }\end{array}$
\end{tabular}

[30] 12 healthy male Malaysian adults 0.49

$0.74^{\text {a }}$

AS only: 1.46
ACT: 3.08

[41] 10 healthy Vietnamese males Half-life: $0.55^{\mathrm{b}}$

[25] 6 healthy adults in Geneva

0.65

Fixed:1.68

Non-fixed:1.42

with mefloquine after washout (Period 2)

Period 1/Day 1:

1.14

Period 1/Day 3:

1.14

Period 2/Day 1:

1.02

Period 2/Day 3:

1.09

\begin{tabular}{ll}
\hline$[23]$ & 15 healthy Cambodian male adults \\
\hline [42] & 12 healthy adults \\
\hline [34] & 86 acute uncomplicated falciparum malaria patients from Malawi and \\
& Gambia
\end{tabular}

$4 \mathrm{mg} / \mathrm{kg}$ once with mefloquine

$1.30^{\mathrm{b}}$

$200 \mathrm{mg}$ once

0.68

Gambia

1, 2, or $4 \mathrm{mg} / \mathrm{kg}$ AS with chlorproguanil and

$1 \mathrm{mg} / \mathrm{kg}: 0.779^{\mathrm{b}}$

dapsone once daily $\times 3$ days

$2 \mathrm{mg} / \mathrm{kg}: 0.917^{\mathrm{b}}$

$4 \mathrm{mg} / \mathrm{kg}: 1.09^{\mathrm{b}}$

\begin{tabular}{llll}
\hline [7] & 12 Vietnamese adult male vivax malaria patients & $100 \mathrm{mg}$ single dose & $0.67(\mathrm{n}=11)$ \\
\hline$[8]$ & 26 Vietnamese adult patients with uncomplicated falciparum malaria & $100 \mathrm{mg}$ single dose & $0.66(\mathrm{n}=16)$
\end{tabular}

[39] 21 children (5 - 13 years) with uncomplicated malaria in Uganda $4 \mathrm{mg} / \mathrm{kg}$ once daily with amodiaquine $\times 3$ days

[38] 24 children with uncomplicated falciparum malaria in Gabon $4 \mathrm{mg} / \mathrm{kg}$ once daily for 3 days in one of two

formulations (blister pack and fixed dose) of AS/ $1.3^{\mathrm{a}}$ mefloquine

Fixed dose: 0.9

$(n=9)^{a}$

Blister pack: 1.0

$(\mathrm{n}=11)^{\mathrm{a}}$

[32] 43 adults with uncomplicated falciparum malaria in Thailand

$200 \mathrm{mg} /$ day for fixed dose AS-mefloquine tablet or Fixed: 1.1 ( $\mathrm{n}=$ $4 \mathrm{mg} / \mathrm{kg} /$ day as nonfixed AS-mefloquine 14)

Nonfixed: 0.8 (n $=18)$

[31] 11 male Thai adults with uncomplicated falciparum malaria

$200 \mathrm{mg}$ AS once, followed by $100 \mathrm{mg} 12$ hours

Acute: $0.64^{a}$

later, then $100 \mathrm{mg}$ once daily $\times 4$ days

Convalescence: $0.66^{\mathrm{a}}$

[43] 24 pregnant Karen women in the $2^{\text {nd }}$ and $3^{\text {rd }}$ trimesters with uncomplicated falciparum malaria

$4 \mathrm{mg} / \mathrm{kg}$ once daily $\times 3$ days with atovaquone plus Half-life: $1.0(\mathrm{n}=$ proguani

$13)^{a}$

PopPK half-life estimate: $1.81 \mathrm{hr}$

[35] 6 male Thai adults with uncomplicated falciparum malaria and 6

$100 \mathrm{mg}$ AS once

Healthy: 0.85 healthy male adults

Patients: 1.06

[9] 8 Vietnamese adults with uncomplicated falciparum malaria and 10 healthy Vietnamese adults

$150 \mathrm{mg}$ once

Healthy: 0.77

Patients: 0.88

[53] $262^{\text {nd }}$ and $3^{\text {rd }}$ trimester pregnant women with asymptomatic falciparum parasitaemia, the same women postpartum, and 25 nonpregnant asymptomatic, parasitaemic controls

$200 \mathrm{mg}$ once

Pregnant: $1.28^{\mathrm{a}}$

Postpartum:1.63 Controls: $1.41^{\mathrm{a}}$

[36] 57 children (2-14 years) with uncomplicated falciparum malaria in $\quad$ AS dose $(\mathrm{mg} / \mathrm{kg})$ Gabon 


\begin{tabular}{|c|c|c|c|}
\hline [37] & $\begin{array}{l}40 \text { children and adults with uncomplicated falciparum malaria in Pailin, } \\
\text { Cambodia and } 40 \text { adults with uncomplicated falciparum malaria in } \\
\text { Wang Pha, Thailand }\end{array}$ & $\begin{array}{l}\text { At each site: } \\
\text { Group 1: AS monotherapy: } 2 \mathrm{mg} / \mathrm{kg} / \text { day } \times 7 \text { days } \\
\text { Group 2: AS } 4 \mathrm{mg} / \mathrm{kg} / \text { day } \times 3 \text { days }+ \text { mefloquine }\end{array}$ & $\begin{array}{l}\text { Thailand: } \\
\text { Group 1: } 0.71^{\mathrm{a}} \\
\text { Group 2: } 0.85^{\mathrm{a}} \\
\text { Cambodia: } \\
\text { Group 1: } 0.84^{\mathrm{a}} \\
\text { Group 2: } 0.77^{\mathrm{a}}\end{array}$ \\
\hline
\end{tabular}

Estimates from PopPK studies are described in the text. Values given as mean unless otherwise specified.

a. Median b. Geometric mean

parent-metabolite model with first-order AS absorption, a one-compartment model for AS and a two-compartment model for DHA. Adjusting for the median weight of the study population $(61.5 \mathrm{~kg})$, the final estimates for AS CL/F and V/F were $19 \mathrm{~L} / \mathrm{kg} / \mathrm{hr}$ and $20 \mathrm{~L} / \mathrm{kg}$, respectively. Similarly adjusting for median weight, DHA central clearance and central volume of distribution were $1.52 \mathrm{~L} / \mathrm{kg} / \mathrm{hr}$ and $1.58 \mathrm{~L} / \mathrm{kg}$, respectively, with weight as a statistically significant covariate on DHA apparent clearance. The only other significant covariate-parameter relationship identified in the model was the effect of food intake on the AS absorption rate constant, with a reduction in absorption rate of $84 \%$ associated with administration of AS with a high fat, high calorie meal. Inter-individual variability was estimated on five of the modelled parameters, with the highest inter-individual variability observed for $\mathrm{Ka}(\% \mathrm{CV}=$ $112 \%)$ and $\mathrm{AS} \mathrm{V/F}(\% \mathrm{CV}=57.4 \%)$.

Stepniewska et al [48] described the PopPK of DHA following oral AS administration in children (6 months - 5 years) with uncomplicated falciparum malaria. AS and DHA pharmacokinetic data were obtained from 70 children who received AS and amodiaquine, but only DHA data could be modelled. Samples were collected once in the first dosing interval and once in the third dosing interval. The authors modelled DHA data using a one-compartment model with first-order input. They estimated DHA CL/F as $0.636 \mathrm{~L} / \mathrm{kg} / \mathrm{hr}$ for the first dosing period, with a substantial additive increase of 0.760 $\mathrm{L} / \mathrm{kg} / \mathrm{hr}$ being associated with the third dosing period. The authors speculated that this modelled increase in clearance reflected pharmacokinetic changes related to resolution of acute illness. DHA apparent volume of distribution, which was not modelled as varying between dosing periods, was estimated as $2.285 \mathrm{~L} / \mathrm{kg}$, with age modelled as a covariate on volume. The authors noted that either age or weight explained a significant portion of the variability on volume, but that the two covariates were not independent. Inter-individual variability was modelled on DHA apparent volume of distribution (\% $\mathrm{CV}=47 \%)$, but no other parameter.

\section{Rectal administration: AS and DHA pharmacokinetics}

The bioavailability of rectally administered AS, as assessed by exposure to DHA, in paediatric patients with moderately severe malaria was estimated to be $23 \%$ in patients administered a dose of $20 \mathrm{mg} / \mathrm{kg}$ and $58 \%$ in patients administered $10 \mathrm{mg} / \mathrm{kg}$ [15]. In one rectal-oral crossover study in healthy volunteers, the mean bioavailability of rectal AS relative to oral AS, as assessed by exposure to DHA, was 54.9\% [42]. However, in a study of similar design, no statistically significant differences in DHA $\mathrm{AUC}_{0-\mathrm{t}}$ following oral and rectal AS administration were observed, although AS AUC was significantly larger and DHA Cmax significantly smaller following rectal, as compared to oral, administration [30]. The inconsistent findings of these two studies may relate to the difficulty of defining a sampling schedule able to optimally capture the unique concentration-time profiles associated with different routes of administration.

Tables 9 and $10[15,30,42,49,50]$ summarize the PK findings of the identified rectal AS administration studies. Tmax for AS following rectal administration occurred on average between 0.58 - 1.43 hours. AS halflife was estimated in only two studies, with half-life estimates of 0.9 - 0.95 hours. These longer half-life estimates may reflect absorption rate-limited elimination of AS. Following rectal administration of AS, DHA concentrations peaked between 1.13 - 2.0 hours, and DHA was eliminated with a half-life averaging $0.79-1.8$ hours. Only one non-PopPK study [15] reported estimates of DHA apparent clearance and volume following rectal AS; those values were $2.6-3.9 \mathrm{~L} / \mathrm{kg} / \mathrm{hr}$ and $4.4-5.9 \mathrm{~L} /$ $\mathrm{kg}$, respectively. As would be expected given that rectal AS administration avoids first-pass metabolism, the discrepancy in AS and DHA AUC values is not as striking with rectal, as compared with oral, administration of AS.

\section{Rectal administration: population pharmacokinetic analyses}

Two population pharmacokinetic analyses of data obtained following rectal AS administration were identified. Simpson et al [51] described the population pharmacokinetics of DHA following rectal AS administration to adult and paediatric patients with moderately severe falciparum malaria. Patients were administered a single dose of $10 \mathrm{mg} / \mathrm{kg}$ AS with follow-up treatment administered orally. AS concentrations could not be successfully modelled. DHA concentrations (424 levels) obtained 
Table 9 Summary of AS pharmacokinetic results following rectal AS administration

\begin{tabular}{|c|c|c|c|c|c|}
\hline Ref. & Subjects \& Regimen & $\begin{array}{l}\mathrm{Cmax} \\
(\mathrm{ng} / \mathrm{mL})\end{array}$ & Tmax (hours) & Half-life (hours) & $\begin{array}{l}\text { AUC } \\
\left(\text { ng*hr/mL) }^{*}\right.\end{array}$ \\
\hline $\begin{array}{l}49] \\
\text { Sirivichay et al, } 2007\end{array}$ & $\begin{array}{l}16 \text { paediatric patients with uncomplicated falciparum malaria } \\
10 \mathrm{mg} / \mathrm{kg}(\mathrm{n}=7) \text { or } 20 \mathrm{mg} / \mathrm{kg}(\mathrm{n}=9) \mathrm{AS} \text { as rectal suppositories }\end{array}$ & $507 ; 561 t^{a}$ & $0.8 ; 1.0^{\mathrm{a}}$ & $0.9 ; 0.9^{a}$ & $692 ; 1076 t^{a}$ \\
\hline $\begin{array}{l}\text { [50] } \\
\text { Halpaap et al, } 1998\end{array}$ & $\begin{array}{l}12 \text { paediatric patients with uncomplicated falciparum malaria } \\
50 \mathrm{mg} \mathrm{AS} \text { as rectal suppository } \\
{[0.86-2.55 \mathrm{mg} / \mathrm{kg} \mathrm{AS}]}\end{array}$ & $90+$ & 0.58 & & \\
\hline $\begin{array}{l}\text { [30] } \\
\text { Navaratnam et al, } 1998\end{array}$ & $\begin{array}{l}12 \text { healthy Malaysian adults } \\
200 \text { mg AS as rectal suppository }\end{array}$ & 448.5 & 1.43 & 0.95 & 796 \\
\hline
\end{tabular}

Estimates from PopPK studies are described in the text. Values given as mean unless otherwise specified.

†Units converted to uniform scale a. Median

from 164 patients were fit to a one-compartment model with fixed, lagged, first-order input (DHA appearance rate: $0.2 / \mathrm{hr}$; lag: $0.14 \mathrm{hr}$ ). Gender and weight were identified as important covariates in the model, with DHA $\mathrm{CL} / \mathrm{F}$ of $3.17 \mathrm{~L} / \mathrm{kg} / \mathrm{hr}$ for males and $2.03 \mathrm{~L} / \mathrm{kg} / \mathrm{hr}$ for females. DHA V/F was estimated as increasing from $1.81 \mathrm{~L} / \mathrm{kg}$ for a $15 \mathrm{~kg}$ subject to $6.34 \mathrm{~L} / \mathrm{kg}$ for a $70 \mathrm{~kg}$ subject. Estimated inter-individual variability was $62 \%$ for $\mathrm{CL} / \mathrm{F}$ and $75 \%$ for $\mathrm{V} / \mathrm{F}$.

Karunajeewa et al [52] conducted population pharmacokinetic analysis of AS and DHA data following administration of $10-15 \mathrm{mg} / \mathrm{kg}$ rectal AS (2 doses, 12 hours apart) to 47 paediatric uncomplicated falciparum or vivax malaria patients in Papua New Guinea. AS data and DHA data were each fit to a one-compartment model; first-order AS absorption was modelled. Due to identifiability concerns, the volume of distribution estimates for AS and DHA were set equal (41.8 L). Weight was an influential covariate on volume. The AS CL/F (mean $\pm \mathrm{SD}$ ) was determined to be $121.2 \pm 35.4 \mathrm{~L} / \mathrm{hr}$ and DHA CL/F to be $44.9 \pm 13.0 \mathrm{~L} / \mathrm{hr}$. Average AS and DHA half-life estimates were 0.27 and 0.71 hours, respectively. The absorption half-life was estimated as 2.3 hours. The model included a bioavailability term for the second dose relative to the first dose $(72 \%)$. The authors conjectured that higher core body temperature when the first dose was administered may have resulted in enhanced rectal blood flow and, therefore, absorption.

Table 10 Summary of DHA pharmacokinetic results following rectal AS administration

\begin{tabular}{|c|c|c|c|c|c|c|c|}
\hline Ref. & Subjects \& Regimen & $\begin{array}{l}\text { Cmax } \\
(\mathrm{ng} / \mathrm{mL})\end{array}$ & $\begin{array}{l}\text { Tmax } \\
\text { (hours) }\end{array}$ & $\begin{array}{l}\text { Clearance } \\
\text { (L/kg/hr) }\end{array}$ & $\begin{array}{l}\text { Volume } \\
\text { (L/kg) }\end{array}$ & $\begin{array}{l}\text { Half-life } \\
\text { (hours) }\end{array}$ & $\begin{array}{l}\text { AUC } \\
\text { (ng*hr/mL) }\end{array}$ \\
\hline $\begin{array}{l}{[49]} \\
\text { Sirivichay et al, } \\
2007\end{array}$ & $\begin{array}{l}16 \text { paediatric patients with uncomplicated falciparum } \\
\text { malaria } \\
10 \mathrm{mg} / \mathrm{kg}(\mathrm{n}=7) \text { or } 20 \mathrm{mg} / \mathrm{kg}(\mathrm{n}=9) \text { AS as rectal } \\
\text { suppositories }\end{array}$ & $\begin{array}{l}898 \\
1535 t^{a}\end{array}$ & $1.5 ; 2.0^{\mathrm{a}}$ & & & $1.3 ; 1.8^{a}$ & $\begin{array}{l}2403 ; \\
5633 十^{a}\end{array}$ \\
\hline $\begin{array}{l}50] \\
\text { Halpaap et al, } \\
1998\end{array}$ & $\begin{array}{l}12 \text { paediatric patients with uncomplicated falciparum } \\
\text { malaria } \\
50 \mathrm{mg} \text { AS as rectal suppository } \\
{[0.86-2.55 \mathrm{mg} / \mathrm{kg} \mathrm{AS}]}\end{array}$ & $180+$ & 1.13 & & & & \\
\hline $\begin{array}{l}{[42]} \\
\text { Awad et al, } \\
2004\end{array}$ & $\begin{array}{l}12 \text { healthy Sudanese adults } \\
200 \text { mg AS as rectal suppository }\end{array}$ & $219.1 \dagger$ & 1.95 & & & 1.21 & 1185.17 \\
\hline $\begin{array}{l}30] \\
\text { Navaratnam et } \\
\text { al, } 1998\end{array}$ & $\begin{array}{l}12 \text { healthy Malaysian adults } \\
200 \text { mg AS as rectal suppository }\end{array}$ & $385.6 \dagger$ & 1.80 & & & & $\begin{array}{l}\mathrm{AUC}_{0-\mathrm{t}} \\
965\end{array}$ \\
\hline $\begin{array}{l}\text { Krishna et al, } \\
2001 \\
{[15]}\end{array}$ & $\begin{array}{l}34 \text { Ghanaian children (8 months - } 7 \text { years) with } \\
\text { moderate falciparum malaria } \\
\text { Group 1: AS } 10 \mathrm{mg} / \mathrm{kg} \text { as rectal suppository, IV AS } 2.4 \\
\text { mg/kg } 12 \mathrm{hr} \text { later } \\
\text { Group 2: AS } 20 \mathrm{mg} / \mathrm{kg} \text { AS as rectal suppository, IV AS } \\
2.4 \mathrm{mg} / \mathrm{kg} 12 \mathrm{hr} \text { later } \\
\text { Group 3: } 2.4 \mathrm{mg} / \mathrm{kg} \text { IV AS, } 20 \mathrm{mg} / \mathrm{kg} \text { AS as rectal } \\
\text { suppository } 12 \mathrm{hr} \text { later }\end{array}$ & $\begin{array}{l}\text { Group 1: } \\
682 t^{a} \\
\text { Group } 2 \& \\
3: \\
881 t^{a}\end{array}$ & $\begin{array}{l}\text { Group 1: } \\
1.7^{\text {a }} \\
\text { Tlag: } \\
0.63^{a} \\
\text { Group } 2 \\
\& 3: \\
1.8^{a} \\
\text { Tlag: } \\
0.37^{a}\end{array}$ & $\begin{array}{l}\text { Group 1: } \\
2.6 \\
\text { Group } 2 \& \\
\text { 3: } \\
3.9\end{array}$ & $\begin{array}{l}\text { Group 1: } \\
4.4^{\mathrm{a}} \\
\text { Group } 2 \& \\
3: \\
5.9^{\mathrm{a}}\end{array}$ & $\begin{array}{l}\text { Group 1: } \\
0.79^{\mathrm{a}} \\
\text { Group } 2 \& \\
\text { 3: } \\
0.85^{\mathrm{a}}\end{array}$ & $\begin{array}{l}\text { Group 1: } \\
2787 \dagger^{a} \\
\text { Group } 2 \& \\
3: \\
3753+^{a}\end{array}$ \\
\hline
\end{tabular}

Estimates from PopPK studies are described in the text. Values given as mean unless otherwise specified

tUnits converted to uniform scale a. Median 


\section{Artesunate and DHA pharmacokinetics in paediatric patients}

The two previously described population pharmacokinetic models describing AS/DHA PK following rectal AS administration were conducted using data from a mixed adult and paediatric population [51] or an exclusively paediatric population [52]; in both of these analyses, weight represented a significant covariate on DHA apparent volume of distribution. In the PopPK analysis of DHA following oral AS administration to young children, Stepniewska et al [48], determined that either weight or age could explain a significant portion of the between subject variability in DHA volume of distribution. These findings suggest that weight, or a highly correlated covariates such as age, is an important predictor variable for DHA PK in paediatric patients. The practice of utilizing AS regimens targeted to a $\mathrm{mg} / \mathrm{kg}$ dosage range should somewhat aid in minimizing weight-based variability in exposure. However, further study would be required in the paediatric population to assess if patient age is an important source of variability beyond that explained by body weight alone. Such study would optimally focus on infants and very young children since the most marked differences in drug metabolism and other physiologic processes would be expected in this patient population.

\section{Artesunate and DHA pharmacokinetics in pregnant women}

Two pharmacokinetic trials have been conducted to characterize AS/DHA pharmacokinetic changes that may be associated with the physiologic changes of pregnancy. McGready et al [43] modelled the PK of DHA following oral administration of AS to $2^{\text {nd }}$ and $3^{\text {rd }}$ trimester pregnant women with acute uncomplicated malaria. Population modelling yielded DHA CL/F and V/F estimates of $1.77 \mathrm{~L} / \mathrm{kg} / \mathrm{hr}$ and $4.63 \mathrm{~L} / \mathrm{kg}$. Non-compartmental analysis of their data yielded estimates of $4.0 \mathrm{~L} / \mathrm{kg} / \mathrm{hr}$ for $\mathrm{CL} / \mathrm{F}$ and $3.4 \mathrm{~L} / \mathrm{kg}$ for $\mathrm{V} / \mathrm{F}$. The authors noted that exposure to DHA following oral AS administration to the pregnant women in the study was substantially lower than that observed in non-pregnant subjects in previous studies.

Onyamboko et al [53] examined the PK of DHA following the oral administration of $200 \mathrm{mg}$ AS to $262^{\text {nd }}$ and $3^{\text {rd }}$ trimester pregnant women with asymptomatic falciparum parasitaemia, the same women 3 months post-partum, and 25 matched asymptomatic parasitaemic female controls in the Democratic Republic of Congo. The median DHA CL/F was $1.39 \mathrm{~L} / \mathrm{kg} / \mathrm{hr}, 1.26$ $\mathrm{L} / \mathrm{kg} / \mathrm{hr}$, and $1.07 \mathrm{~L} / \mathrm{kg} / \mathrm{hr}$ for pregnant, post-partum, and non-pregnant control subjects, respectively. Median DHA V/F was $2.84 \mathrm{~L} / \mathrm{kg}$ for pregnant, $3.00 \mathrm{~L} / \mathrm{kg}$ for post-partum, and $2.45 \mathrm{~L} / \mathrm{kg}$ for non-pregnant control subjects. DHA AUC was significantly different (geometric mean ratio: $0.68,90 \% \mathrm{CI}: 0.57-0.81$ ) for the pregnant as compared to control subjects; however, DHA AUC values for pregnant women and the same women at three months post-partum were relatively similar. A population pharmacokinetic analysis [54] of the AS and DHA data from pregnant and control women in the Onyamboko et al study modelled the data using mixed-order absorption with a one-compartment model for AS and a one-compartment model for DHA; in that analysis, pregnancy was associated with a significant increase in DHA CL/F, as well as a trend towards increased volume of distribution.

\section{Artemisinin resistance in Plasmodium falciparum malaria}

The recent emergence in western Cambodia of $P$. falciparum with reduced susceptibility to artemisinin derivatives has been the source of substantial concern; questions regarding the relationship between the pharmacokinetics of these derivatives and the observed delayed parasite clearance times have been posed. Dondorp et al [37] assessed the efficacy of two regimens of AS for uncomplicated falciparum malaria at a site in western Cambodia, where reduced susceptibility was expected, and another in northwestern Thailand, where substantially reduced susceptibility was not anticipated. These regimens consisted of $2 \mathrm{mg} / \mathrm{kg} /$ day oral AS monotherapy $\times 7$ days or $4 \mathrm{mg} / \mathrm{kg} /$ day oral AS $\times 3$ days followed by two doses of mefloquine. As expected, patients in Cambodia displayed significantly longer parasite clearance times as compared to patients in Thailand. However, no apparent clinically relevant differences in AS and DHA pharmacokinetics were observed between the two study sites. Additionally, no relationship between measures of AS or DHA exposure and parasite clearance time was observed. These results suggest that the observed reduced artemisinin susceptibility of $P$. falciparum in western Cambodia is not highly sensitive to PK parameters for AS and DHA within the $2-4 \mathrm{mg} /$ $\mathrm{kg} /$ day AS dosage range.

\section{Effect of infection status on artesunate and DHA pharmacokinetics}

Multiple studies have attempted to investigate and characterize any changes in AS and DHA PK associated with malaria infection. Two of the studies described above, conducted by Stepniewska et al [48] and Karbwang et al [31], determined that the PK of orally administered AS may differ in the acute stage of infection as compared to the convalescent stage. Stepniewska et al [48] determined that DHA clearance was substantially lower on the first day of treatment as compared to the third day. Karbwang et al [31] determined that DHA Cmax was significantly decreased, and AUC not 
significantly changed, on the first day of treatment as compared to the fifth day. On the first day of treatment, higher AS clearance was also reported. Newton et al [19] used bioassay data to investigate the anti-malarial activity in patients with falciparum malaria during the patients' acute and convalescent phases. The analysis indicated that anti-malarial activity AUC and Cmax were two-fold higher in the acute phase as compared to the convalescent phase for subjects administered the same dose of oral AS. Correspondingly, apparent clearance and volume of distribution of anti-malarial activity were significantly smaller in the acute phase of infection. Although these three studies do not fully align regarding the effect of disease resolution on AS/DHA PK, perhaps due to the use of differing populations, sampling time points, and time course of sampling, taken together these studies do suggest that some alteration in PK may occur over the course of treatment. It should be noted that changes over the course of treatment are likely not due to time-dependency of AS or DHA PK, as has been observed with various other artemisinin derivatives; following oral administration of AS over a typical treatment course, time-dependent kinetics are not apparent [55].

A direct comparison of healthy and parasitaemic subjects was conducted by Teja-Isavadharm et al [35], who studied the PK of DHA following oral AS administration to six healthy adults and six adult falciparum malaria patients. The investigators determined that AUC and Cmax of DHA were significantly higher in subjects with malaria as compared to healthy subjects. Binh et al [9] obtained similar results when comparing the PK in eight patients with falciparum malaria and ten healthy subjects. However, given the relatively small size of both the Binh et al [9] and Teja-Isavadharm et al [35] studies, drawing definitive conclusions regarding differences in PK between healthy and infected subjects is not possible at present. Nonetheless, as DHA clearance is dependent upon hepatic blood flow, a reduction in clearance, and consequently an increase in exposure, associated with acute infection would be consistent with DHA's known pharmacokinetic properties.

\section{Drug-drug interactions}

Given the metabolic pathways of AS (esterase-catalyzed hydrolysis) and DHA (UGT-mediated conjugation), AS should not be susceptible to the many common drugdrug interactions involving CYP450 enzymes. Agents evaluated for their drug interaction potential with orally administered AS include atovaquone-proguanil [56], sulphadoxine-pyrimethamine [57], pyronaridine [47], mefloquine [24], chlorproguanil-dapsone [34], artemisinin [41], and amodiaquine [27]. AS coadministration does not appear to alter the PK of atovaquone-proguanil
[56] or sulphadoxine-pyrimethamine [57]. No significant change in DHA AUC was detected when AS was coadministered with mefloquine [24]. In the PopPK analysis of AS and DHA PK following oral AS administration by Tan $e t$ al (described above), coadministration of AS with the Mannich-base derivative pyronaridine was not determined to exert a significant influence on AS or DHA pharmacokinetics [47]. Multiple dose administration of AS did not alter the PK of artemisinin; however, artemisinin coadministration with AS in ten healthy adults was associated with a more than two-fold increase in DHA AUC, a finding which led the authors to speculate that artemisinin may act as a UGT inhibitor [41]. Finally, AS coadministration with chlorproguanil-dapsone did not produce significant alterations in chlorproguanil or dapsone $\mathrm{PK}$, although moderate increases in exposure to the metabolites chlorcycloguanil and monoacetyl dapsone were detected. No clinically significant alterations of AS and DHA pharmacokinetics were found to be associated with AS-chlorproguanil-dapsone combination therapy [34].

Orrell et al [27] investigated the drug interaction potential of artesunate and amodiaquine. The authors conducted a crossover study in which 12 healthy African adults received $4 \mathrm{mg} / \mathrm{kg}$ AS on day 0 and either amodiaquine or amodiaquine+AS on day 7 , with the alternative regimen administered on day 28 . The investigators determined that when amodiaquine and AS were coadministered, the mean DHA AUC was approximately $33 \%$ lower, the mean DHA Cmax was $49 \%$ lower, and the mean DHA half-life was 57\% longer than when AS was administered alone [27]. The AUC of the amodiaquine metabolite desethylamodiaquine was determined to be $45 \%$ lower when amodiaquine was coadministered with AS. However, the subject with the highest desethylamodiaquine AUC during amodiaquine+AS coadministration was excluded from the amodiaquine drug interaction analysis [27]. Orrell et al do not speculate on the source of the interaction. Given the small size of the study, and the lack of any clear physiologic basis for the observed interaction, further study would be needed to fully characterize this potential drug-drug interaction.

\section{Conclusion}

AS is a clinically versatile artemisinin derivative utilized for the treatment of mild to severe malaria infection. Given the therapeutic significance of AS, and the necessity of appropriate AS dosing, substantial research has been performed investigating the pharmacokinetics of AS and its active metabolite DHA. The results of the studies identified in this review indicate that administration of IV AS produces an AS Cmax of substantially greater magnitude than observed with any other route of administration. Following IV administration, AS 
hydrolysis to DHA occurs rapidly, producing DHA peak concentrations within 25 minutes post-dose. AS and DHA display average clearance values of $2-3 \mathrm{~L} / \mathrm{kg} / \mathrm{hr}$ and $0.5-1.5 \mathrm{~L} / \mathrm{kg} / \mathrm{hr}$, respectively, with volume estimates averaging $0.1-0.3 \mathrm{~L} / \mathrm{kg}$ for AS and $0.5-1.0 \mathrm{~L} / \mathrm{kg}$ for DHA. IM administration of AS is associated with high bioavailability, as assessed by DHA exposure. Although generally displaying similar PK to IV AS, IM AS does produce lower Cmax, higher V/F, and longer half-life values for AS, as well as longer Tmax values for DHA, than IV administration.

Following oral AS administration, peak AS concentrations are attained within an hour, with AS eliminated with a half-life of 20 - 45 minutes. DHA Cmax values occur within two hours post-dose; DHA half-life values average 0.5 - 1.5 hours. A marked discrepancy in AS and DHA AUC values is apparent following oral AS administration, with DHA AUC values commonly determined to be more than 10-fold higher than corresponding AS AUC values. The PK parameters obtained in studies with rectal AS administration are generally similar to those obtained in studies with oral administration, although AS Tmax is delayed and AS half-life extended. PopPK analyses of AS/DHA data following oral and rectal AS administration suggest that weight and pregnancy represent influential predictors of DHA pharmacokinetics following AS administration.

To date, drug interactions studies of AS with various other anti-malarial agents have not yielded strong evidence of clinically relevant drug-drug interactions involving AS. Several relatively small studies examining the effects of infection on AS and DHA PK indicate that acute malaria infection may be associated with PK changes; however, determining the exact nature of such changes will require further study. Similarly, present evidence suggests that pregnancy may result in PK changes which will require further study for full elucidation.

\footnotetext{
Author details

'Pharmaceutical Sciences and Experimental Therapeutics, University of lowa College of Pharmacy, 115 South Grand Avenue, lowa City, IA 52242, USA. ${ }^{2}$ Medicines for Malaria Venture, International Center Cointrin, 20 route de Pré-Bois, 1216 Cointrin, Geneva, Switzerland. ${ }^{3}$ Pharmaceutical Research Services, 1300 Seaport Blvd, Suite 500, Redwood City, California 94063, USA. ${ }^{4}$ Shin Poong Pharmaceuticals, 748-31, Yoksam-Dong, Kangnam-Ku, Seoul 135-925, Republic of Korea.
}

\section{Authors' contributions \\ CAM, SD, IBF, DJ, CS and LF all made substantial contributions to the conception, organization, and revision of the review. All of the authors critically reviewed the manuscript and approved the final version for submission.}

\section{Competing interests}

Chang-Sik Shin is an employee of Shin Poong Pharmaceuticals.

Received: 21 June 2011 Accepted: 13 September 2011 Published: 13 September 2011

\section{References}

1. World Health Organization: Guidelines for the treatment of malaria. 2 edition Geneva, Switzerland: World Health Organization; 2011, Rev. 1.

2. Lindegardh $N$, Hanpithakpong W, Kamanikom B, Singhasivanon $P$, Socheat D, Yi P, Dondorp AM, McGready R, Nosten F, White NJ, Day NP: Major pitfalls in the measurement of artemisinin derivatives in plasma in clinical studies. J Chromatogr B Analyt Technol Biomed Life Sci 2008, 876:54-60

3. Li Q, Xie LH, Haeberle A, Zhang J, Weina P: The evaluation of radiolabeled artesunate on tissue distribution in rats and protein binding in humans. Am J Trop Med Hyg 2006, 75:817-826.

4. Xie LH, Li Q, Zhang J, Weina PJ: Pharmacokinetics, tissue distribution and mass balance of radiolabeled dihydroartemisinin in male rats. Malar $\mathrm{J}$ 2009, 8:112.

5. Batty KT, llett KF, Davis TM: Protein binding and alpha: beta anomer ratio of dihydroartemisinin in vivo. Br J Clin Pharmacol 2004, 57:529-533.

6. Batty KT, lletr KE, Powell SM, Martin J, Davis TM: Relative bioavailability of artesunate and dihydroartemisinin: investigations in the isolated perfused rat liver and in healthy Caucasian volunteers. Am J Trop Med Hyg 2002, 66:130-136.

7. Batty KT, Le AT, llett KF, Nguyen PT, Powell SM, Nguyen CH, Truong XM, Vuong VC, Huynh VT, Tran QB, Nguyen VM, Davis TM: A pharmacokinetic and pharmacodynamic study of artesunate for vivax malaria. Am J Trop Med Hyg 1998, 59:823-827.

8. Batty KT, Thu LT, Davis TM, llett KF, Mai TX, Hung NC, Tien NP, Powell SM, Thien HV, Binh TQ, Kim NV: A pharmacokinetic and pharmacodynamic study of intravenous vs oral artesunate in uncomplicated falciparum malaria. Br J Clin Pharmacol 1998, 45:123-129.

9. Binh TQ, llett KF, Batty KT, Davis TM, Hung NC, Powell SM, Thu LT, Thien HV, Phuong $\mathrm{HL}$, Phuong VD: Oral bioavailability of dihydroartemisinin in Vietnamese volunteers and in patients with falciparum malaria. $\mathrm{Br} J \mathrm{Clin}$ Pharmacol 2001, 51:541-546.

10. Ilett KF, Batty KT, Powell SM, Binh TQ, Thu le TA, Phuong HL, Hung NC, Davis TM: The pharmacokinetic properties of intramuscular artesunate and rectal dihydroartemisinin in uncomplicated falciparum malaria. $\mathrm{Br} J$ Clin Pharmacol 2002, 53:23-30.

11. Li Q, Cantilena LR, Leary KJ, Saviolakis GA, Miller RS, Melendez V, Weina PJ: Pharmacokinetic profiles of artesunate after single intravenous doses at $0.5,1,2,4$, and $8 \mathrm{mg} / \mathrm{kg}$ in healthy volunteers: a phase I study. Am J Trop Med Hyg 2009, 81:615-621.

12. Nealon C, Dzeing A, Muller-Romer U, Planche T, Sinou V, Kombila M, Kremsner PG, Parzy D, Krishna S: Intramuscular bioavailability and clinical efficacy of artesunate in gabonese children with severe malaria. Antimicrob Agents Chemother 2002, 46:3933-3939.

13. Newton PN, Barnes Kl, Smith PJ, Evans AC, Chierakul W, Ruangveerayuth R, White NJ: The pharmacokinetics of intravenous artesunate in adults with severe falciparum malaria. Eur J Clin Pharmacol 2006, 62:1003-1009.

14. Davis TM, Phuong HL, llett KF, Hung NC, Batty KT, Phuong VD, Powell SM, Thien HV, Binh TQ: Pharmacokinetics and pharmacodynamics of intravenous artesunate in severe falciparum malaria. Antimicrob Agents Chemother 2001, 45:181-186.

15. Krishna S, Planche T, Agbenyega T, Woodrow C, Agranoff D, Bedu-Addo G, Owusu-Ofori AK, Appiah JA, Ramanathan S, Mansor SM, Navaratnam V: Bioavailability and preliminary clinical efficacy of intrarectal artesunate in Ghanaian children with moderate malaria. Antimicrob Agents Chemother 2001, 45:509-516.

16. Hess KM, Goad JA, Arguin PM: Intravenous artesunate for the treatment of severe malaria. Ann Pharmacother 2010, 44:1250-1258.

17. Gautam A, Ahmed T, Batra V, Paliwal J: Pharmacokinetics and pharmacodynamics of endoperoxide antimalarials. Curr Drug Metab 2009, 10:289-306.

18. Ilett KF, Ethell BT, Maggs $J$, Davis TM, Batty KT, Burchell B, Binh TQ, Thu le TA, Hung NC, Pirmohamed M, Park BK, Edwards G: Glucuronidation of dihydroartemisinin in vivo and by human liver microsomes and expressed UDP-glucuronosyltransferases. Drug Metab Dispos 2002, 30:1005-1012.

19. Newton P, Suputtamongkol Y, Teja-Isavadharm P, Pukrittayakamee S, Navaratnam V, Bates I, White N: Antimalarial bioavailability and disposition of artesunate in acute falciparum malaria. Antimicrob Agents Chemother 2000, 44:972-977. 
20. Ittarat W, Looareesuwan S, Pootrakul P, Sumpunsirikul P, Vattanavibool $P$, Meshnick SR: Effects of alpha-thalassemia on pharmacokinetics of the antimalarial agent artesunate. Antimicrob Agents Chemother 1998, 42:2332-2335

21. Hien TT, Davis TM, Chuong LV, llett KF, Sinh DX, Phu NH, Agus C, Chiswell GM, White NJ, Farrar J: Comparative pharmacokinetics of intramuscular artesunate and artemether in patients with severe falciparum malaria. Antimicrob Agents Chemother 2004, 48:4234-4239.

22. Olliaro PL, Nair NK, Sathasivam K, Mansor SM, Navaratnam V: Pharmacokinetics of artesunate after single oral administration to rats. BMC Pharmacol 2001, 1:12.

23. Chanthap L, Tsuyuoka R, Na-Bangchang K, Nivanna N, Suksom D, Sovannarith T, Socheat D: Investigation of bioavailability, pharmacokinetics and safety of new pediatric formulations of artesunate and mefloquine. Southeast Asian J Trop Med Public Health 2005, 36:34-43.

24. Davis TM, England M, Dunlop AM, Page-Sharp M, Cambon N, Keller TG, Heidecker $J L$, llett KF: Assessment of the effect of mefloquine on artesunate pharmacokinetics in healthy male volunteers. Antimicrob Agents Chemother 2007, 51:1099-1101

25. Benakis A, Paris M, Loutan L, Plessas CT, Plessas ST: Pharmacokinetics of artemisinin and artesunate after oral administration in healthy volunteers. Am J Trop Med Hyg 1997, 56:17-23.

26. Navaratnam V, Ramanathan S, Wahab MS, Siew Hua G, Mansor SM, Kiechel JR, Vaillant M, Taylor WR, Olliaro P: Tolerability and pharmacokinetics of non-fixed and fixed combinations of artesunate and amodiaquine in Malaysian healthy normal volunteers. Eur J Clin Pharmacol 2009, 65:809-821.

27. Orrell C, Little F, Smith P, Folb P, Taylor W, Olliaro P, Barnes Kl: Pharmacokinetics and tolerability of artesunate and amodiaquine alone and in combination in healthy volunteers. Eur J Clin Pharmacol 2008, 64:683-690.

28. Na-Bangchang K, Karbwang J, Congpoung K, Thanavibul A, Ubalee R: Pharmacokinetic and bioequivalence evaluation of two generic formulations of oral artesunate. Eur J Clin Pharmacol 1998, 53:375-376.

29. Diem Thuy LT, Ngoc Hung L, Danh PT, Na-Bangchang K: Absence of timedependent artesunate pharmacokinetics in healthy subjects during 5day oral administration. Eur J Clin Pharmacol 2008, 64:993-998.

30. Navaratnam V, Mansor SM, Mordi MN, Akbar A, Abdullah MN: Comparative pharmacokinetic study of oral and rectal formulations of artesunic acid in healthy volunteers. Eur J Clin Pharmacol 1998, 54:411-414.

31. Karbwang J, Na-Bangchang K, Congpoung K, Thanavibul A, Harinasuta T: Pharmacokinetics of oral artesunate in thai patients with uncomplicated falciparum malaria. Clin Drug Investig 1998, 15:37-43.

32. Krudsood S, Looareesuwan S, Tangpukdee N, Wilairatana P, Phumratanaprapin W, Leowattana W, Chalermrut K, Ramanathan S, Navaratnam V, Olliaro P, Vaillant M, Kiechel JR, Taylor WR: New fixed-dose artesunate-mefloquine formulation against multidrug-resistant Plasmodium falciparum in adults: a comparative phase Ilb safety and pharmacokinetic study with standard-dose nonfixed artesunate plus mefloquine. Antimicrob Agents Chemother 2010, 54:3730-3737.

33. Sinou V, Malaika LT, Taudon N, Lwango R, Alegre SS, Bertaux L, Sugnaux F, Parzy D, Benakis A: Pharmacokinetics and pharmacodynamics of a new ACT formulation: Artesunate/Amodiaquine (TRIMALACT) following oral administration in African malaria patients. Eur J Drug Metab Pharmacokinet 2009, 34:133-142.

34. Miller AK, Bandyopadhyay N, Wootton DG, Duparc S, Kirby PL, Winstanley PA, Ward SA: Pharmacokinetics of chlorproguanil, dapsone, artesunate and their major metabolites in patients during treatment of acute uncomplicated Plasmodium falciparum malaria. Eur J Clin Pharmacol 2009, 65:977-987.

35. Teja-Isavadharm P, Watt G, Eamsila C, Jongsakul K, Li Q, Keeratithakul G, Sirisopana N, Luesutthiviboon L, Brewer TG, Kyle DE: Comparative pharmacokinetics and effect kinetics of orally administered artesunate in healthy volunteers and patients with uncomplicated falciparum malaria. Am J Trop Med Hyg 2001, 65:717-721.

36. Ramharter M, Kurth F, Schreier AC, Nemeth J, Glasenapp I, Belard S, Schlie M, Kammer J, Koumba PK, Cisse B, Mordmuller B, Lell B, Issifou S, Oeuvray C, Fleckenstein L, Kremsner PG: Fixed-dose pyronaridineartesunate combination for treatment of uncomplicated falciparum malaria in pediatric patients in Gabon. J Infect Dis 2008, 198:911-919.
37. Dondorp AM, Nosten F, Yi P, Das D, Phyo AP, Tarning J, Lwin KM, Ariey F, Hanpithakpong W, Lee SJ, Ringwald P, Silamut K, Imwong M, Chotivanich K, Lim P, Herdman T, An SS, Yeung S, Singhasivanon P, Day NP, Lindegardh N, Socheat $D$, White $\mathrm{NJ}$ : Artemisinin resistance in Plasmodium falciparum malaria. N Engl J Med 2009, 361:455-467.

38. Ramharter M, Kurth FM, Belard S, Bouyou-Akotet MK, Mamfoumbi MM, Agnandji ST, Missinou MA, Adegnika AA, Issifou S, Cambon N, Heidecker JL, Kombila M, Kremsner PG: Pharmacokinetics of two paediatric artesunate mefloquine drug formulations in the treatment of uncomplicated falciparum malaria in Gabon. J Antimicrob Chemother 2007, 60:1091-1096.

39. Mwesigwa J, Parikh S, McGee B, German P, Drysdale T, Kalyango JN, Clark TD, Dorsey G, Lindegardh N, Annerberg A, Rosenthal PJ, Kamya MR, Aweeka F: Pharmacokinetics of artemether-lumefantrine and artesunateamodiaquine in children in Kampala, Uganda. Antimicrob Agents Chemother 2010, 54:52-59.

40. Na-Bangchang K, Krudsood S, Silachamroon U, Molunto P, Tasanor O, Chalermrut K, Tangpukdee N, Matangkasombut O, Kano S, Looareesuwan S: The pharmacokinetics of oral dihydroartemisinin and artesunate in healthy Thai volunteers. Southeast Asian J Trop Med Public Health 2004, 35:575-582.

41. Zhang SQ, Hai TN, llett KF, Huong DX, Davis TM, Ashton M: Multiple dose study of interactions between artesunate and artemisinin in healthy volunteers. Br J Clin Pharmacol 2001, 52:377-385.

42. Awad MI, Eltayeb IB, Baraka OZ, Behrens RH, Alkadru AM: Pharmacokinetics of artesunate following oral and rectal administration in healthy Sudanese volunteers. Trop Doct 2004, 34:132-135.

43. McGready R, Stepniewska K, Ward SA, Cho T, Gilveray G, Looareesuwan S, White NJ, Nosten F: Pharmacokinetics of dihydroartemisinin following oral artesunate treatment of pregnant women with acute uncomplicated falciparum malaria. Eur J Clin Pharmacol 2006, 62:367-371.

44. Teja-Isavadharm P, Watt G, Eamsila C, Jongsakul K, Li Q, Keeratithakul G, Sirisopana N, Luesutthiviboon L, Brewer TG, Kyle DE: Comparative pharmacokinetics and effect kinetics of orally administered artesunate in healthy volunteers and patients with uncomplicated falciparum malaria. Am J Trop Med Hyg 2001, 65:717-721.

45. Newton PN, van Vugt M, Teja-Isavadharm P, Siriyanonda D, Rasameesoroj M, Teerapong P, Ruangveerayuth $R$, Slight T, Nosten F, Suputtamongkol Y, Looareesuwan S, White NJ: Comparison of oral artesunate and dihydroartemisinin antimalarial bioavailabilities in acute falciparum malaria. Antimicrob Agents Chemother 2002, 46:1125-1127.

46. Bethell DB, Teja-Isavadharm P, Cao XT, Pham TT, Ta TT, Tran TN, Nguyen TT, Pham TP, Kyle D, Day NP, White NJ: Pharmacokinetics of oral artesunate in children with moderately severe Plasmodium falciparum malaria. Trans R Soc Trop Med Hyg 1997, 91:195-198.

47. Tan B, Naik H, Jang IJ, Yu KS, Kirsch LE, Shin CS, Craft JC, Fleckenstein L. Population pharmacokinetics of artesunate and dihydroartemisinin following single- and multiple-dosing of oral artesunate in healthy subjects. Malar J 2009, 8:304

48. Stepniewska K, Taylor W, Sirima SB, Ouedraogo EB, Ouedraogo A, Gansane A, Simpson JA, Morgan CC, White NJ, Kiechel JR: Population pharmacokinetics of artesunate and amodiaquine in African children. Malar J 2009, 8:200.

49. Sirivichayakul C, Sabchareon A, Pengsaa K, Thaiarporn I, Chaivisuth A, NaBangchang K, Wisetsing P, Chanthavanich P, Pojjaroen-Anant C: Comparative study of the effectiveness and pharmacokinetics of two rectal artesunate/oral mefloquine combination regimens for the treatment of uncomplicated childhood falciparum malaria. Ann Trop Paediatr 2007, 27:17-24.

50. Halpaap B, Ndjave M, Paris M, Benakis A, Kremsner PG: Plasma levels of artesunate and dihydroartemisinin in children with Plasmodium falciparum malaria in Gabon after administration of 50-milligram artesunate suppositories. Am J Trop Med Hyg 1998, 58:365-368.

51. Simpson JA, Agbenyega T, Barnes KI, Di Perri G, Folb P, Gomes M, Krishna S, Krudsood S, Looareesuwan S, Mansor S, Mcllleron H, Miller R, Molyneux M, Mwenechanya J, Navaratnam V, Nosten F, Olliaro P, Pang L, Ribeiro I, Tembo M, van Vugt M, Ward S, Weerasuriya K, Win K, White NJ: Population pharmacokinetics of artesunate and dihydroartemisinin following intrarectal dosing of artesunate in malaria patients. PLoS Med 2006, 3:e444.

52. Karunajeewa HA, llett KF, Dufall K, Kemiki A, Bockarie M, Alpers MP, Barrett PH, Vicini P, Davis TM: Disposition of artesunate and dihydroartemisinin after administration of artesunate suppositories in 
children from Papua New Guinea with uncomplicated malaria. Antimicrob Agents Chemother 2004, 48:2966-2972.

53. Onyamboko MA, Meshnick SR, Fleckenstein L, Koch MA, Atibu J, Lokomba V, Douoguih M, Hemingway-Foday J, Wesche D, Ryder RW, Bose C, Wright LL, Tshefu AK, Capparelli EV: Pharmacokinetics and pharmacodynamics of artesunate and dihydroartemisinin following oral treatment in pregnant women with asymptomatic Plasmodium falciparum infections in Kinshasa DRC. Malar J 2011, 10:49.

54. Morris CA, Onyamboko MA, Capparelli E, Koch MA, Atibu J, Lokomba V, Douoguih M, Hemingway-Foday J, Wesche D, Ryder RW, Bose C, Wright L, Tshefu AK, Meshnick S, Fleckenstein L: Population pharmacokinetics of artesunate and dihydroartemisinin in pregnant and non-pregnant women with malaria. Malar J 2011, 10:114.

55. Diem Thuy LT, Ngoc Hung L, Danh PT, Na-Bangchang K: Absence of timedependent artesunate pharmacokinetics in healthy subjects during 5day oral administration. Eur J Clin Pharmacol 2008, 64:993-998.

56. Van Vugt M, Edstein MD, Proux S, Lay K, Ooh M, Looareesuwan S, White NJ, Nosten F: Absence of an interaction between artesunate and atovaquone-proguanil. Eur J Clin Pharmacol 1999, 55:469-474.

57. Minzi OM, Gupta A, Haule AF, Kagashe GA, Massele AY, Gustafsson LL: Lack of impact of artesunate on the disposition kinetics of sulfadoxine/ pyrimethamine when the two drugs are concomitantly administered. Eur J Clin Pharmacol 2007, 63:457-462.

doi:10.1186/1475-2875-10-263

Cite this article as: Morris et al:: Review of the clinical pharmacokinetics of artesunate and its active metabolite dihydroartemisinin following intravenous, intramuscular, oral or rectal administration. Malaria Journal 2011 10:263.

\section{Submit your next manuscript to BioMed Central and take full advantage of:}

- Convenient online submission

- Thorough peer review

- No space constraints or color figure charges

- Immediate publication on acceptance

- Inclusion in PubMed, CAS, Scopus and Google Scholar

- Research which is freely available for redistribution

Submit your manuscript at www.biomedcentral.com/submit 